\title{
Article
}

\section{The bZIP Transcription Factor HapX Is Post-Translationally Regulated to Control Iron Homeostasis in Aspergillus fumigatus}

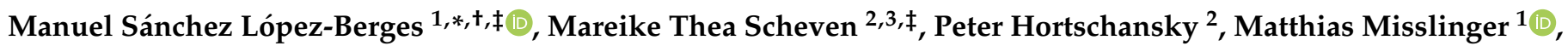

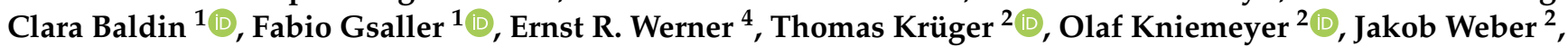 \\ Axel A. Brakhage ${ }^{2,3, *}$ and Hubertus Haas $1, *$ (D)
}

check for updates

Citation: López-Berges, M.S.; Scheven, M.T.; Hortschansky, P.; Misslinger, M.; Baldin, C.; Gsaller, F.; Werner, E.R.; Krüger, T.; Kniemeyer,

O.; Weber, J.; et al. The bZIP

Transcription Factor HapX Is

Post-Translationally Regulated to

Control Iron Homeostasis in Aspergillus fumigatus. Int. J. Mol. Sci. 2021, 22, 7739. https://doi.org/ $10.3390 /$ ijms22147739

Academic Editors: Andrea Battistoni and Serena Ammendola

Received: 5 July 2021

Accepted: 15 July 2021

Published: 20 July 2021

Publisher's Note: MDPI stays neutral with regard to jurisdictional claims in published maps and institutional affiliations.

Copyright: (c) 2021 by the authors. Licensee MDPI, Basel, Switzerland. This article is an open access article distributed under the terms and conditions of the Creative Commons Attribution (CC BY) license (https:// creativecommons.org/licenses/by/ $4.0 /)$.
1 Institute of Molecular Biology, Biocenter, Medical University of Innsbruck, 6020 Innsbruck, Austria; matthias.misslinger@i-med.ac.at (M.M.); clara.baldin@i-med.ac.at (C.B.); fabio.gsaller@i-med.ac.at (F.G.)

2 Department of Molecular and Applied Microbiology, Leibniz Institute for Natural Product Research and Infection Biology (Leibniz-HKI), 07745 Jena, Germany; Mareike.Scheven@web.de (M.T.S.); Peter.Hortschansky@hki-jena.de (P.H.); Thomas.Krueger@hki-jena.de (T.K.); Olaf.Kniemeyer@hki-jena.de (O.K.); jakob.weber.de@gmail.com (J.W.)

3 Institute of Microbiology, Friedrich Schiller University Jena, 07743 Jena, Germany

4 Division of Biological Chemistry, Biocenter, Innsbruck Medical University, 6020 Innsbruck, Austria; ernst.r.werner@i-med.ac.at

* Correspondence: ge2snlpm@uco.es (M.S.L.-B.); axel.brakhage@leibniz-hki.de (A.A.B.); hubertus.haas@i-med.ac.at (H.H.)

+ Current address: Departamento de Genética, Universidad de Córdoba, 14071 Córdoba, Spain.

$\ddagger$ These authors contributed equally to this work.

Abstract: The airborne fungus Aspergillus fumigatus causes opportunistic infections in humans with high mortality rates in immunocompromised patients. Previous work established that the bZIP transcription factor HapX is essential for virulence via adaptation to iron limitation by repressing iron-consuming pathways and activating iron acquisition mechanisms. Moreover, HapX was shown to be essential for transcriptional activation of vacuolar iron storage and iron-dependent pathways in response to iron availability. Here, we demonstrate that HapX has a very short half-life during iron starvation, which is further decreased in response to iron, while siderophore biosynthetic enzymes are very stable. We identified Fbx22 and SumO as HapX interactors and, in agreement, HapX post-translational modifications including ubiquitination of lysine $\mathrm{e}^{161}$, sumoylation of lysine $\mathrm{e}^{242}$ and phosphorylation of threonine $\mathrm{e}^{319}$. All three modifications were enriched in the immediate adaptation from iron-limiting to iron-replete conditions. Interfering with these post-translational modifications, either by point mutations or by inactivation, of Fbx22 or SumO, altered HapX degradation, heme biosynthesis and iron resistance to different extents. Consistent with the need to precisely regulate HapX protein levels, overexpression of hapX caused significant growth defects under iron sufficiency. Taken together, our results indicate that post-translational regulation of HapX is important to control iron homeostasis in A. fumigatus.

Keywords: iron homeostasis; HapX; Aspergillus fumigatus; post-translational regulation

\section{Introduction}

Fungi are an extremely adaptable class of microorganisms mostly comprised of saprophytes thriving on dead organic material. Nevertheless, a relatively small number of fungal species have evolved a parasitic lifestyle associated with the ability to cause disease in both animals and plants. Among them, around 400 fungal species have been reported as pathogens of mammals [1] with Aspergillus fumigatus being one of the most common pathogenic molds in humans [2]. In addition to non-invasive forms of aspergillosis, patients with compromised immune system are at high risk of developing invasive aspergillosis. Today, A. fumigatus belongs to the most prevalent airborne fungal pathogens of humans 
with estimated 3,000,000 cases of chronic pulmonary aspergillosis and 250,000 cases of lifethreatening invasive aspergillosis worldwide $[3,4]$. The lack of early and specific diagnosis and limited therapeutic options aggravate this problem, resulting in a case fatality rate of invasive aspergillosis between 30-90\% [3,5]. Considering the rapid evolution of pathogenic fungi [6] and the emergence of resistant strains to currently available treatments [7,8], there is an urgent need for the development of new antifungals, a process that requires a comprehensive knowledge of the cellular mechanisms underlying pathogenicity.

Iron is an essential element for almost every organism since it serves as a cofactor of many cellular processes; however, its excess can be highly toxic to the cell by promoting the production of reactive oxygen species $[9,10]$. Consequently, iron homeostatic mechanisms are indispensable to guarantee the optimal balance of this metal. In order to understand these dynamic processes, and because adapting to iron limitation has been shown to be essential for fungal pathogenicity in both animal and plant hosts [11-15], several genome-wide transcriptional analyses have been conducted in different fungi over the last $10-15$ years $[11,12,14,16]$. Like other filamentous fungi, A. fumigatus responds to iron limitation by upregulating iron acquisition mechanisms, such as reductive iron assimilation (RIA) and siderophore-mediated iron uptake, and downregulating iron-consuming pathways, including heme biosynthesis and respiration. This adaptation mainly relies on the bZIP transcription factor HapX and, importantly, it is essential for virulence of A. fumigatus in a murine model of invasive aspergillosis [11]. Moreover, HapX was also shown to be required for the adaptation to iron excess, mainly via activating vacuolar iron deposition [17]. The dual role of HapX is based on its ability to sense iron via interaction with the monothiol glutaredoxin GrxD and [2Fe-2S] cluster binding $[18,19]$. hapX is highly transcribed during iron starvation, while its expression is repressed by iron [11,20].

At the mechanistic level, HapX functions via physical interaction with the CCAATbinding complex (CBC) [20-22] and contains the following domains (Figure 1): A " $b(\mathrm{ZIP})$ " basic and a "coiled-coil" domain mediating DNA-binding, an N-terminal Hap4-like domain mediating interaction with the $\mathrm{CBC}$, and four cysteine rich regions (CRR A-D), which are involved in iron sensing [17]. However, to date, it is unknown whether HapX is additionally regulated at the protein level to control iron homeostasis in A. fumigatus.

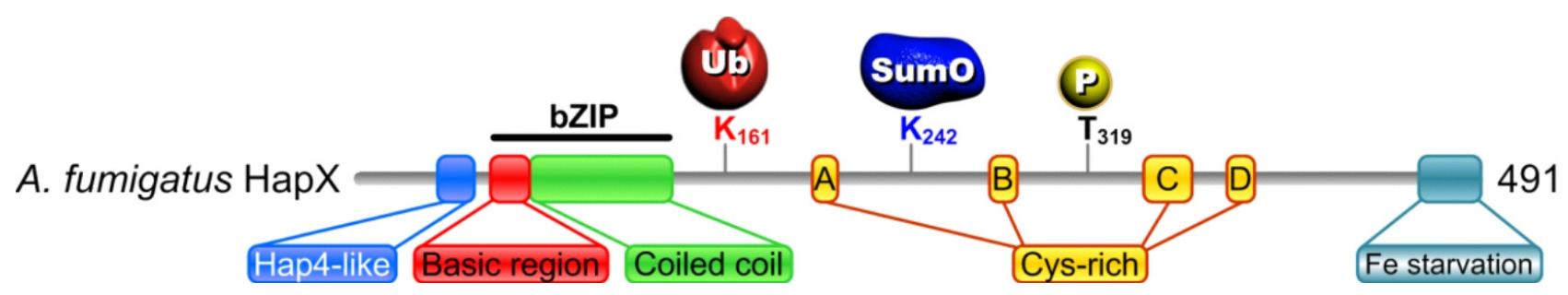

Figure 1. Schematic representation of the A. fumigatus HapX domain organization. The N-terminal Hap4-like CBC binding domain (CBC-BD) is shown in blue. Basic region and coiled coil domains of the bZIP domain are depicted in red and green, respectively. Cysteine-rich regions are marked in yellow. Cysteine-rich regions A and B are essential for HapX function during iron excess but not during iron starvation, while the C-terminus, shown in turquoise, is crucial during iron starvation. Post-translational modifications of residues K161 (ubiquitination), K242 (sumoylation) and T319 (phosphorylation), objects of this study, are shown in dark red, blue and yellow, respectively.

In the current study, we characterized HapX protein regulation during the short-term adaptation from iron-limiting to iron-replete conditions, showing that HapX is quickly degraded throughout this fast adaptation. We identified relevant HapX interactors, such as Fbx22 and SumO, and accordingly, several HapX post-translational modifications, like ubiquitination of lysine 161, sumoylation of lysine 242 and phosphorylation of threonine 319. Disturbing these post-translational modifications affected HapX degradation, heme biosynthesis and iron resistance to different extents. Additionally, in agreement with the necessity to regulate HapX protein levels, overexpression of hapX produced significant growth defects under iron sufficiency. Collectively, our results demonstrate that post- 
translational modification of HapX is important for the maintenance of iron homeostasis in A. fumigatus.

\section{Results}

2.1. HapX Is Rapidly Degraded during Adaptation from Iron-Depleted to Iron-Replete Conditions in a Proteasome Dependent Manner

Iron starvation causes extensive transcriptional changes in fungi [11-13]. In A. fumigatus, like in other filamentous fungi, many of these changes are mediated by HapX [11,17] and have been studied in detail over the past two decades [23]. In order to study HapX at the protein level, we harvested A. fumigatus wild-type strain after growth in media reflecting iron starvation $(-\mathrm{Fe})$, iron sufficiency $(+\mathrm{Fe})$, and short-term adaptation from $-\mathrm{Fe}$ to $+\mathrm{Fe}$ (shift iron, $\mathrm{sFe}$ ), i.e., $60 \mathrm{~min}$ after adding iron to iron-starved cultures. The quality of the samples, with regard to iron status, was tested by real time qRT-PCR of well-known iron-regulated genes: (i) induced in -Fe conditions, such as hapX itself, mirB (encoding a siderophore transporter), sidF and sidH (encoding enzymes required for extracellular siderophore biosynthesis); and (ii) induced in sFe and $+\mathrm{Fe}$ conditions, such as sre $A$ (encoding a transcription factor required for iron uptake repression), $c y c A$ (encoding the cytochrome $c$ ), $c c c A$ (encoding a vacuolar iron transporter), and hem $A$ (encoding a heme biosynthesis protein). Transcript levels of all the tested genes were as expected according to $[11,16,17,20]$ (Figure S1A). For protein detection, we used a rabbit polyclonal antiserum raised against a peptide encompassing A. fumigatus HapX amino acid residues 161 to 491 [18]. Consistent with the expression of the gene, HapX is highly produced in -Fe but not in + Fe steady state cultures. Remarkably, we observed that most of the HapX signal was gone in the sFe condition sample, indicating that HapX is quickly degraded during the adaptation of A. fumigatus from iron-depletion to iron-sufficiency (Figure 2A).

To further investigate this regulation, we performed a sFe experiment analyzing samples collected in $-\mathrm{Fe}$ and 15, 30 and $60 \mathrm{~min}$ after the iron shift. Around 70\% of HapX is degraded within the first $15 \mathrm{~min}$ demonstrating that the degradation process is fast (Figure 2B). Interestingly, HapX migrates as a band of higher molecular mass at this time point, suggesting that the protein is post-translationally modified after iron supplementation. The amount of HapX detected 30 and 60 min after the iron shift is even lower, about $15 \%$ of that found in -Fe; however, in these samples HapX mobility was not increased (Figure 2B). Next, we measured transcript levels of hapX and showed that most of its mRNA was lost $15 \mathrm{~min}$ after the iron shift slightly increasing at later time points (Figure S1B). For comparison, we tested protein stability of SidF and SidH, enzymes required for the biosynthesis of A. fumigatus extracellular siderophores fusarinine $C(\mathrm{FsC})$ and triacetylfusarinine $C$ (TAFC) [24,25], whose coding genes are transcriptionally co-regulated with hapX (Figures S1 and S2A). Detection of the two enzymes, using Venus-tagged strains denoted ${ }^{v}$ sidF and ${ }^{v}$ sidH (see material and methods) showed that, in contrast to HapX, proteins remained highly stable during the $1 \mathrm{~h}$ sFe experiment (Figure S2B).

Considering the fast degradation of HapX during sFe conditions, we tested whether the proteasome could be involved in this process. To address this question, we conducted a sFe experiment with and without the selective proteasome inhibitor MG132 [26] and showed that supplementation of this compound significantly reduced HapX degradation compared to the solvent control DMSO (Figure 2C).

Taken together, these results show that HapX is rapidly degraded in A. fumigatus during short-term adaptation from iron starvation to iron-replete conditions in a proteasome dependent manner. 
A
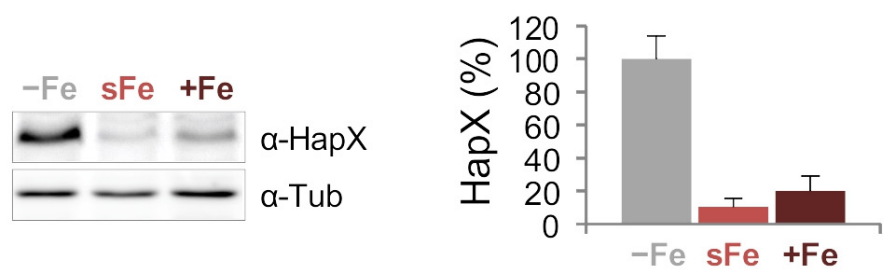

B
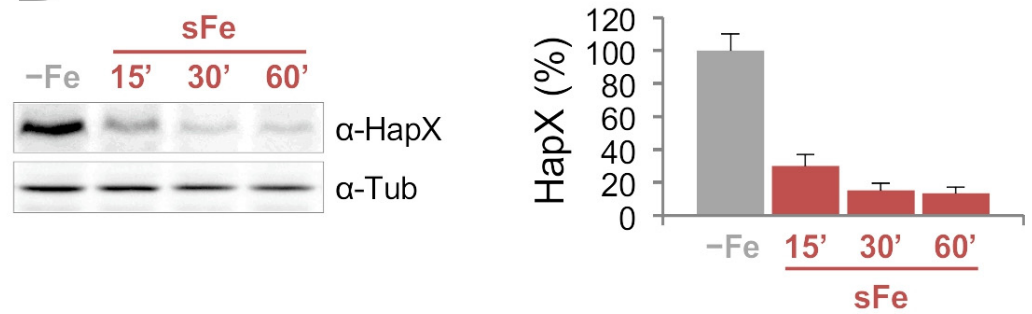

C
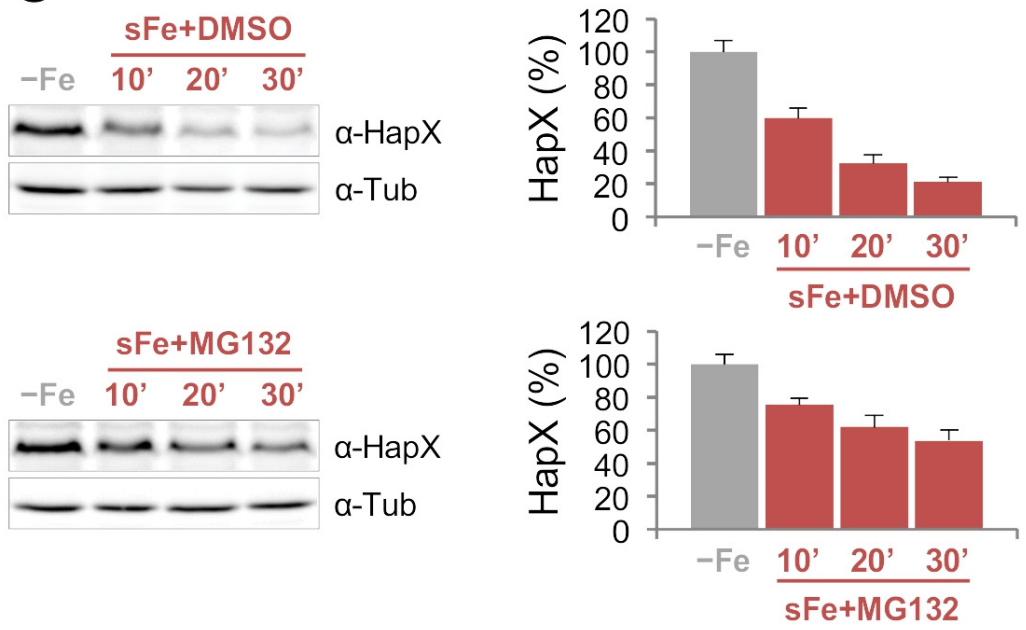

Figure 2. HapX is rapidly degraded during the adaptation from iron-depleted to iron-replete conditions in a proteasome dependent manner. (A-C). HapX quantification by Western blot analysis. Samples were obtained from the wild-type strain grown at $37^{\circ} \mathrm{C}$ in liquid Aspergillus Minimal Medium (AMM) $-\mathrm{Fe}(20 \mathrm{~h}$ in iron depletion), $\mathrm{sFe}(20 \mathrm{~h}$ in iron depletion and then supplemented with $10 \mu \mathrm{M} \mathrm{FeSO}_{4}$ for $\left.1 \mathrm{~h}\right)$ and $+\mathrm{Fe}\left(20 \mathrm{~h}\right.$ in $\left.30 \mu \mathrm{M} \mathrm{FeSO}_{4}\right)(\mathbf{A})$; grown at $37{ }^{\circ} \mathrm{C}$ in $\mathrm{AMM}-\mathrm{Fe}$ and $\mathrm{sFe}$ for 15, 30 and $60 \mathrm{~min}(\mathbf{B})$; or grown at $37^{\circ} \mathrm{C}$ in $\mathrm{AMM}-\mathrm{Fe}$ and sFe for 10, 20 and $30 \mathrm{~min}$ with or without $50 \mu \mathrm{M}$ MG132, dissolved in DMSO (C). Left panels: Representative Western blot analysis showing HapX protein levels. $\alpha$-Tubulin was used as loading control. Right panels: Densitometric protein quantification. HapX protein levels were normalized to Tubulin and expressed relative to those in $-\mathrm{Fe}$. Bars represent standard deviations from two independent biological experiments with two technical replicates each.

\subsection{HapX Turnover Is Very Fast during Iron Limitation}

To study HapX turnover rate under iron-limiting conditions, we analyzed its half-life by culturing the wild-type strain in $-\mathrm{Fe}$ conditions before adding the translation inhibitor cycloheximide (chx) [27], and taking samples 15, 30 and $60 \mathrm{~min}$ after the addition of the compound. Protein quantification showed that HapX levels decreased rapidly after chx treatment (Figure 3A); however, importantly, in contrast to the sFe experiment, the mobility of the protein remained unaltered during the whole experiment (Figure 3B). These results indicate that HapX turnover rate is extremely fast during iron limitation independently of the modifications seen under sFe conditions. 
A

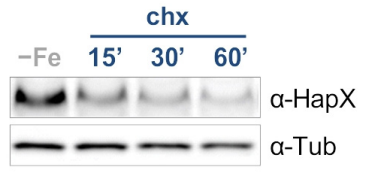

B

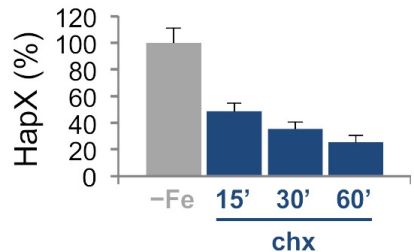

Figure 3. HapX turnover is very fast in - Fe conditions. (A,B). HapX quantification by Western blot analysis. Samples were obtained from the wild-type strain grown at $37{ }^{\circ} \mathrm{C}$ in $\mathrm{AMM}-\mathrm{Fe}$ and then supplemented with $50 \mu \mathrm{g} \cdot \mathrm{mL}^{-1}$ cycloheximide (chx) for 15, 30 and $60 \mathrm{~min}(\mathrm{~A})$. Left panel: Representative Western blot analysis showing HapX protein levels. $\alpha$-Tubulin was used as loading control. Right panel: Densitometric protein quantification. HapX protein levels were normalized to Tubulin and expressed relative to those in -Fe. Bars represent standard deviations from two independent biological experiments with two technical replicates each. (B). Comparison of sFe (Figure 1B) and chx (Figure 3A) samples separated in an 7.5\% SDS-acrylamide gel for a better band size resolution.

\subsection{HapX Affinity Purification Studies Identified Protein Interactors and Post-Translational Modifications during the Adaptation from Iron-Deficiency to Iron-Replete Conditions}

To study the HapX interactome and possible protein post-translational modifications taking place specifically during the adaptation from iron-depleted to iron-replete conditions, we cultured $A$. fumigatus wild-type strain and ${ }^{m} h a p X$, a strain producing an $\mathrm{N}$-terminal Myc-tagged HapX version (see material and methods), under sFe conditions and collected samples in -Fe and 10, 20 and 30 min after the iron shift. Subsequently, the corresponding crude cell extracts were subjected to Myc-Trap affinity purification [28]. Here, the wild-type was used as a negative control to discriminate unspecifically binding proteins. Importantly, ${ }^{m}$ hapX is phenotypically indistinguishable from the wild-type strain (Figure S3A). Effective enrichment of ${ }^{\mathrm{M}}$ HapX was validated by Western blot and nLC-MS/MS analysis (Figure S3B,C). Eluates from three independent biological Myc-Trap experiments were digested either with a Trypsin/LysC mixture or GluC proteases and subsequently analyzed by nLC-MS/MS.

\subsubsection{HapX Specifically Interacts with Fbx22 and SumO during sFe Conditions}

For visualization of specific HapX-interacting proteins, label-free quantification (LFQ) abundances of the most enriched proteins identified in ${ }^{m}$ hapX Myc-Trap eluates were represented as volcano plots, displaying the enrichment of a given protein vs. its reproducibility in the three replicates, in comparison with the LFQ abundances in wild-type control eluates (Figure 4). As previously reported [18], and regardless of the digestion used after Myc-Trap affinity purification, we identified the monothiol glutaredoxin GrxD as one of the most highly enriched proteins under iron limitation (Figure 4A). GrxD, known to be essential for sensing iron starvation in A. fumigatus [18], was also detected, in lower abundance, in the three time points after the iron shift (Figure 4B-D). In addition to GrxD, we identified two previously uncharacterized interactors related to protein post-translational modification. The F-box protein Fbx22 (Afu6g13030) and the small ubiquitin-related modifier SumO (Afu1g10850) co-immunoprecipitated with ${ }^{{ }^{M}}$ HapX to different extents (Figure 4). F-box proteins, as part of the SCF complex (Skp, Cullin, F-box containing complex) [29,30], mediate the ubiquitination of proteins destined for the proteasomal degradation or other regulatory processes [31,32]. Fbx22 was co-purified during early time points after the iron shift and significantly co-enriched when the Myc-Trap eluates were digested with GluC (Figure 4B,C). On the other hand, SumO, a member of the small ubiquitin-like protein 
family of around $10 \mathrm{kDa}$ able to modulate localization, activity, stability and interactions of the target proteins [33], was significantly enriched with independence of the digestion performed in $-\mathrm{Fe}$ conditions and, and with higher abundance, at early time points after the iron shift (Figure $4 \mathrm{~A}-\mathrm{C}$ ).
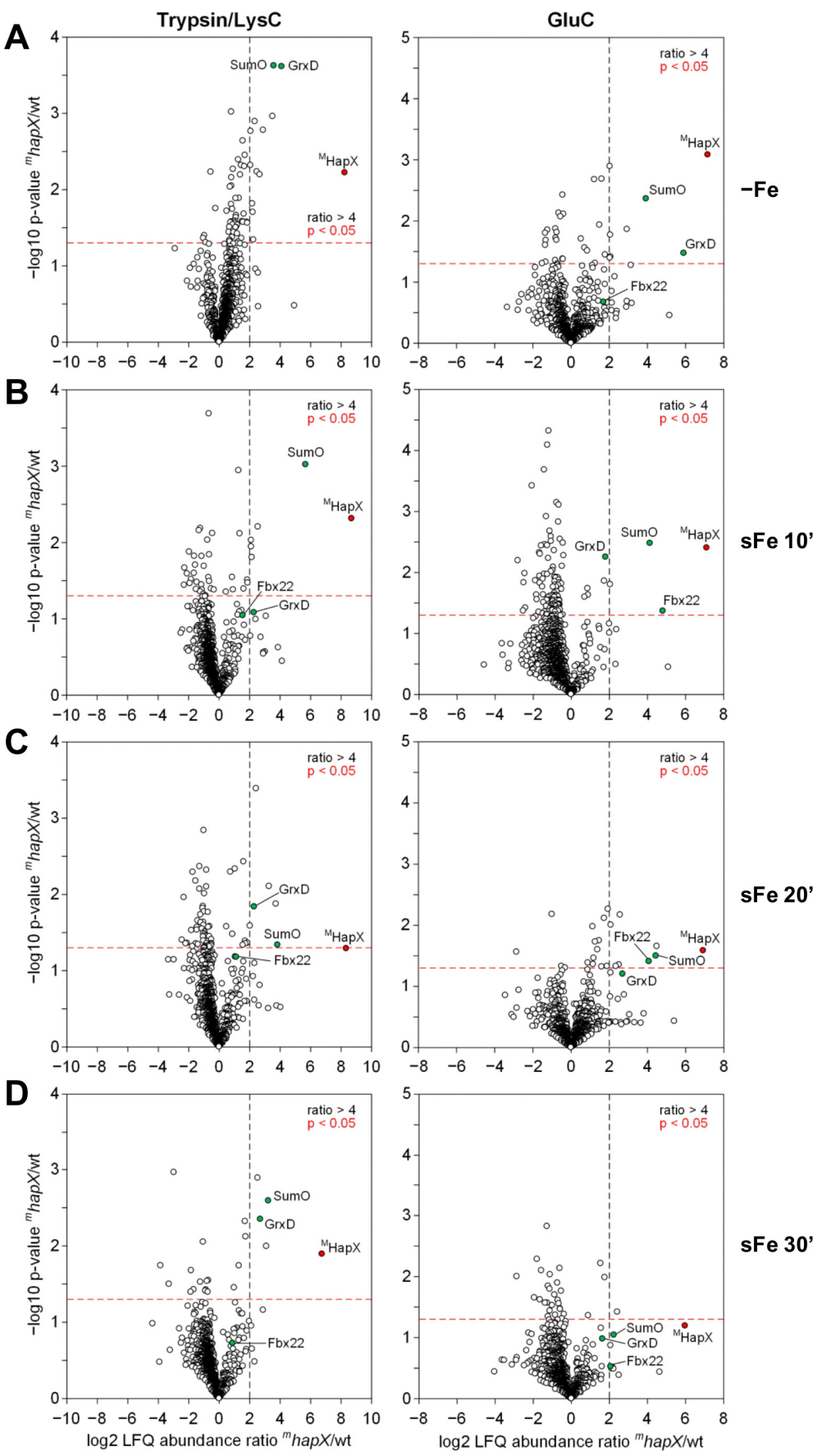

Figure 4. HapX physically interacts with Fbx22 and SumO during the adaptation from irondepleted to iron-replete conditions. (A-D). Volcano-plot visualization of specifically enriched proteins interacting with ${ }^{\mathrm{M}} \mathrm{HapX}$. Absolute label-free quantification (LFQ) abundances of proteins were determined by nLC-MS/MS analysis after Myc-Trap affinity purification from A. fumigatus ${ }^{m}$ hapX and plotted against LFQ abundances in wild-type eluates. Samples were obtained growing the strains at $37{ }^{\circ} \mathrm{C}$ in AMM - Fe (A) and sFe 10 (B), 20 (C) and 30 (D) min after the iron shift. Each dot represents the enrichment of a given protein vs. its reproducibility in three biological replicates. Specifically co-purified proteins are indicated by green dots. The bait, ${ }^{\mathrm{M}} \mathrm{HapX}$, is marked as a red dot. 
Collectively, these results show that HapX interacts with partners involved in posttranslational modification related to protein degradation and reinforces our previous finding of the proteasome implicated in HapX degradation.

Inactivation of Fbx22 and SumO Affects Iron Resistance, HapX Levels and Degradation and Heme Biosynthesis

To study the role of Fbx22 and SumO in A. fumigatus HapX degradation, we generated $f b x 22 \Delta$ and sum $O \Delta$ strains replacing the entire coding sequences with the pyrithiamine resistance gene (see Material and Methods). To determine the impact of both mutations on growth during different levels of iron availability, we cultured $f b x 22 \Delta$ and sumO $\Delta$, in comparison with the wild-type strain, in solid media under different iron conditions. $f b x 22 \Delta$ displayed no major phenotypical changes while, as shown for other Aspergilli [33-35], sum $\mathrm{O} \Delta$ showed a small decrease in radial growth both under $-\mathrm{Fe}$ and $+\mathrm{Fe}$ conditions. Despite this, interestingly, sum $\Delta \Delta$ and to a lesser degree $f b x 22 \Delta$ were slightly more resistant than the wild-type strain to highly toxic iron concentrations (hFe) (Figure 5A). Quantification of HapX levels during A. fumigatus adaptation from iron-depleted to iron-replete conditions showed that protein degradation was slightly less efficient in both mutants, with a difference somewhat higher in $f b x 22 \Delta$ (Figure 5B, see in comparison with Figure 2B). Importantly, the HapX band of higher molecular mass observed 15 min after the iron shift in the wild-type strain is still seen in both mutants (Figure 5B). Furthermore, we observed that -Fe HapX levels were increased about 1.75-fold and 3-fold in fbx22 $\Delta$ and sumO $\Delta$, respectively (Figure 5C). Since heme biosynthesis is de-repressed in hapX $\Delta$ under $-\mathrm{Fe}$ conditions [11,20], accumulation of its iron-free precursor protoporphyrin IX (PpIX) can be used as a readout of HapX activity under - Fe conditions. In line, PpIX biosynthesis, known to be increased in hapX $\Delta[11,20]$, decreased both in fbx22 $\Delta$ and sumO $\Delta$ (Figure 5D). These results show that Fbx22 and SumO are important factors in the regulation of HapX protein levels, influencing both the production of important iron-related metabolites and iron resistance.

\subsubsection{HapX Is Ubiquitinated, Sumoylated and Phosphorylated during sFe Conditions}

Considering the identified HapX interactome, we analyzed affinity-purified HapX peptides for ubiquitination and sumoylation signals. When Myc-Trap purified eluates were digested with the Trypsin/LysC mixture, a peptide containing $\mathrm{K}_{161}(\mathrm{GG})$, a ubiquitin remnant, was co-purified (Figure $6 \mathrm{~A}$ ). Vice versa, a peptide containing $\mathrm{K}_{242}(\mathrm{QIGG})$, a sumoylation remnant, was co-enriched when the eluates were digested with GluC (Figure 6B). Indeed, K242 was in silico identified as part of a negatively charged amino acid-dependent SUMOylation motif (NDSM) present in HapX $\left({ }^{241}\right.$ IKPDPEEMEID $^{251}$ ) [36]. Importantly, both modifications, like the interactions of HapX with Fbx22 and SumO, were co-enriched with the highest abundance $10 \mathrm{~min}$ after the iron shift (Figure 6A,B). In addition to these modifications, we identified several HapX peptides with phosphorylated residues (not shown); however, among them, only threonine (T) 319 was found phosphorylated in a relatively high proportion and differentially between $-\mathrm{Fe}$ vs. sFe conditions. Quantification of phosphorylated vs. non-phosphorylated peptides revealed that T319 phosphorylation was highly induced during sFe conditions (Figure $6 \mathrm{C}$ ). These results suggest that the HapX post-translational modifications found during the adaptation of A. fumigatus from iron starvation to iron-sufficiency (shown in Figure 1) could be relevant in the regulation of the protein degradation process. 
A

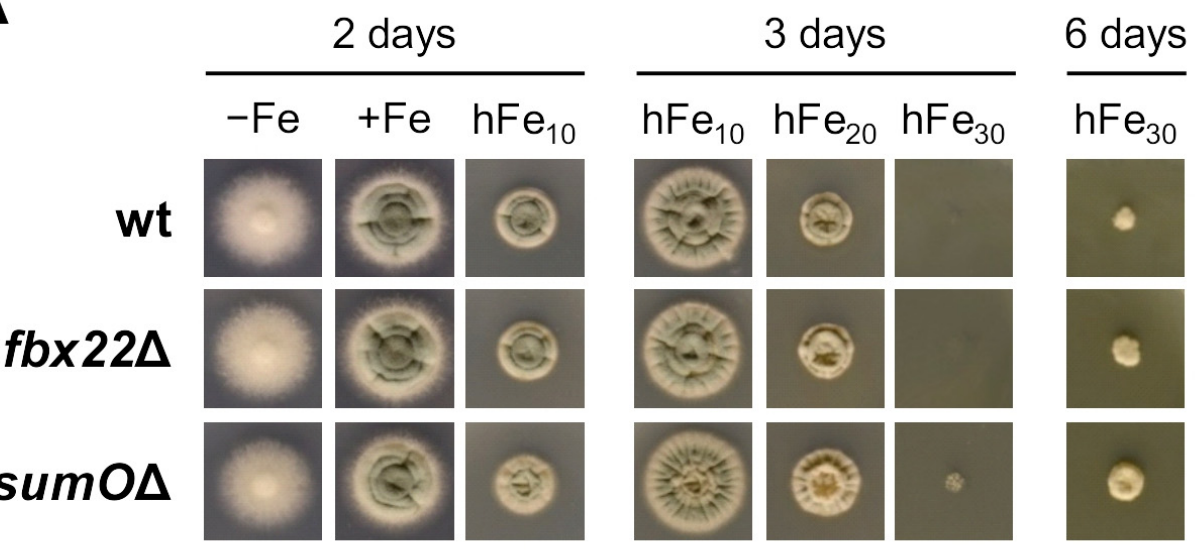

B

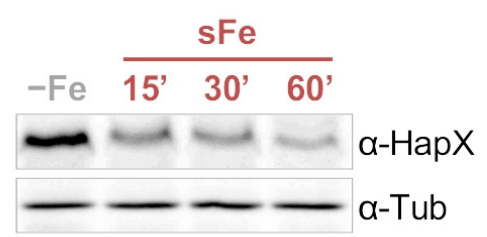

$f b \times 22 \Delta$

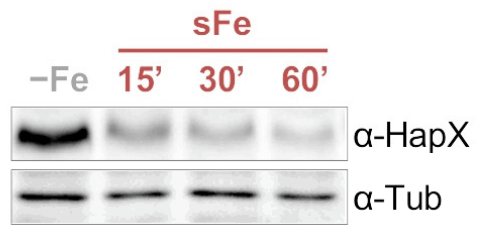

sumOA
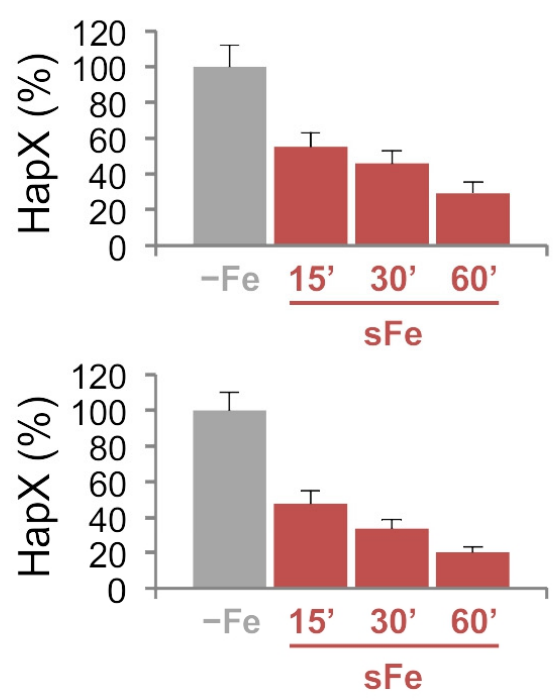

C

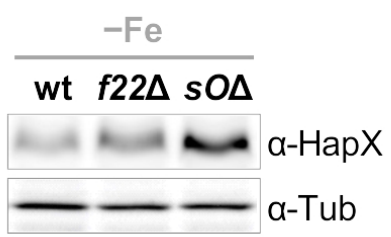

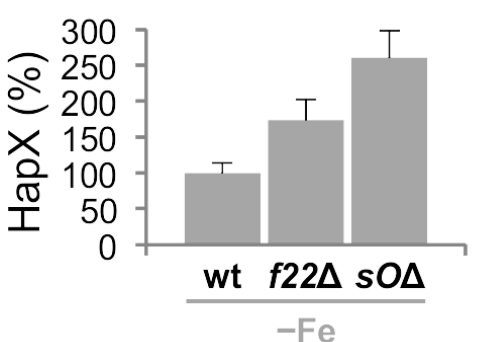

D

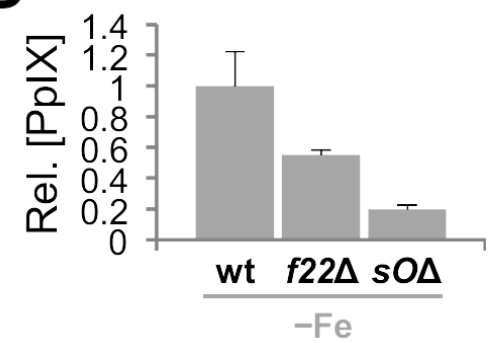

Figure 5. SumO and Fbx22 are important for iron resistance, regulation of HapX protein levels and heme biosynthesis. (A). Growth of the indicated strains on solid AMM - Fe (iron depletion), +Fe (30 $\left.\mu \mathrm{M} \mathrm{FeSO}_{4}\right)$, and hFe (10, 20 or $30 \mathrm{mM}$ $\mathrm{FeSO}_{4}$ ). Plates were incubated for the indicated time periods at $37^{\circ} \mathrm{C}$. Note that wild-type (wt) left panel is identical to that in Figure S3A. It is shown again here for clarity. (B,C). HapX quantification by Western blot analysis. Samples were obtained from the indicated strains grown under sFe conditions as in Figure 2B (B); or under - Fe conditions as in Figure 2A (C). Left panels: Representative Western blot analysis showing HapX protein levels. $\alpha$-Tubulin was used as loading control. Right panels: Densitometric protein quantification. HapX protein levels were normalized to Tubulin and expressed relative to those in -Fe. (D). Quantification of PpIX in -Fe conditions. PpIX levels were normalized to the amount of total protein and expressed relative to those in the wild-type strain. Bars represent standard deviations from two independent biological experiments with two technical replicates each. 
A

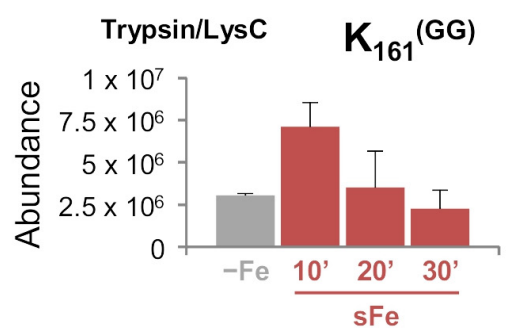

B

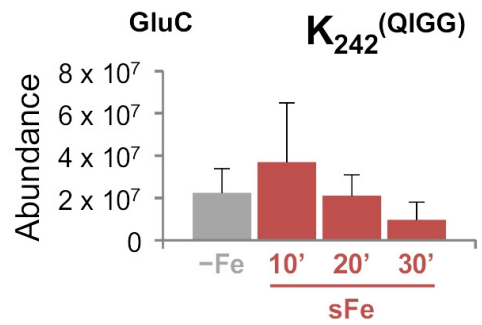

C
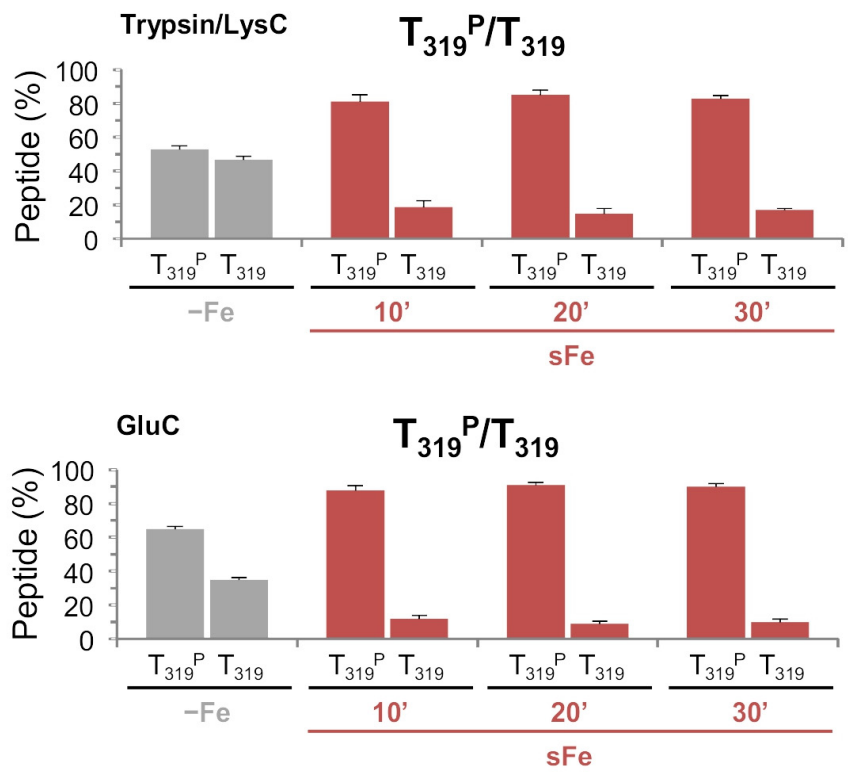

Figure 6. Ubiquitination of K161, sumoylation of K242, and phosphorylation of $\mathrm{T} 319$ are induced during a shift from iron-depleted to iron-replete conditions. (A-C) Label-free quantification of K161 ubiquitination (A), HapX K242 sumoylation (B), and T319 phosphorylation (C) determined by nLCMS/MS analysis after Myc-Trap affinity purification from crude extracts of ${ }^{m}$ hapX grown in AMM -Fe and then supplemented with $10 \mu \mathrm{M} \mathrm{FeSO}_{4}$ for 10, 20 and $30 \mathrm{~min}$ (sFe). Eluates were digested as indicated. Bars represent standard deviations from three independent biological experiments.

Point Mutations in HapX K161, K242 and T319 Affect Iron Resistance, HapX Levels and Degradation and Heme Biosynthesis

To analyze the role of K161 ubiquitination, K242 sumoylation and T319 phosphorylation in HapX post-translational regulation, we generated the corresponding point-mutated strains taking advantage of the availability of the basic plasmid phap $\mathrm{X}^{\mathrm{R}}$-hph, containing the hapX coding sequence C-terminally linked with the S-tag under the control of the native hapX promoter and terminator regions (see materials and methods) [17]. Therefore, our reference here is hap $X^{S}$, a strain producing a C-terminal S-tagged HapX version showing no phenotypical differences with the wild-type strain (compare Figures 5A and 7). K161 and K242 were substituted by arginine (R), thus preventing ubiquitination and sumoylation, respectively; while T319 was replaced by the non-phosphorylatable alanine (A). We denoted these strains hap $X^{K 161 R}$, hap $X^{K 242 R}$ and hap $X^{T 319 A}$. Moreover, we generated all possible double mutant combinations, including hap $X^{K 161 R-K 242 R}$, hap $X^{K 161 R-T 319 A}$ and hap $\mathrm{X}^{K 242 R-T 319 A}$. Unlike the recipient strain hapX $\Delta$ [11], all the mutants showed no major growth defects on solid media under different iron conditions, indicating that these HapX versions are functional (Figure 7). Consistent with the phenotypes observed in $f b x 22 \Delta$ and sumOs (Figure 5A), hapX $X^{K 161 R}$ and hapX $X^{K 242 R}$ were slightly more resistant than the reference strain to $\mathrm{hFe}$ concentrations; however, in contrast, hap $\mathrm{X}^{\mathrm{T} 319 \mathrm{~A}}$ was more sensitive 
(Figure 7). Interestingly, we showed that the phenotype of the double mutants was, in all cases, determined by the combination of each of the single mutations. Note that hFe resistance is: (1) amplified in hap $X^{K 161 R-K 242 R}$ in comparison with any of the single mutants, (2) increased in hap $X^{K 161 R-T 319 A}$ and hapX $X^{K 242 R-T 319 A}$ in comparison with hapX $X^{T 319 A}$, and (3) decreased in hap $X^{K 161 R-T 319 A}$ and hap $X^{K 242 R-T 319 A}$ in comparison with hap $X^{K 161 R}$ and hap $X^{K 242 R}$, respectively (Figure 7).

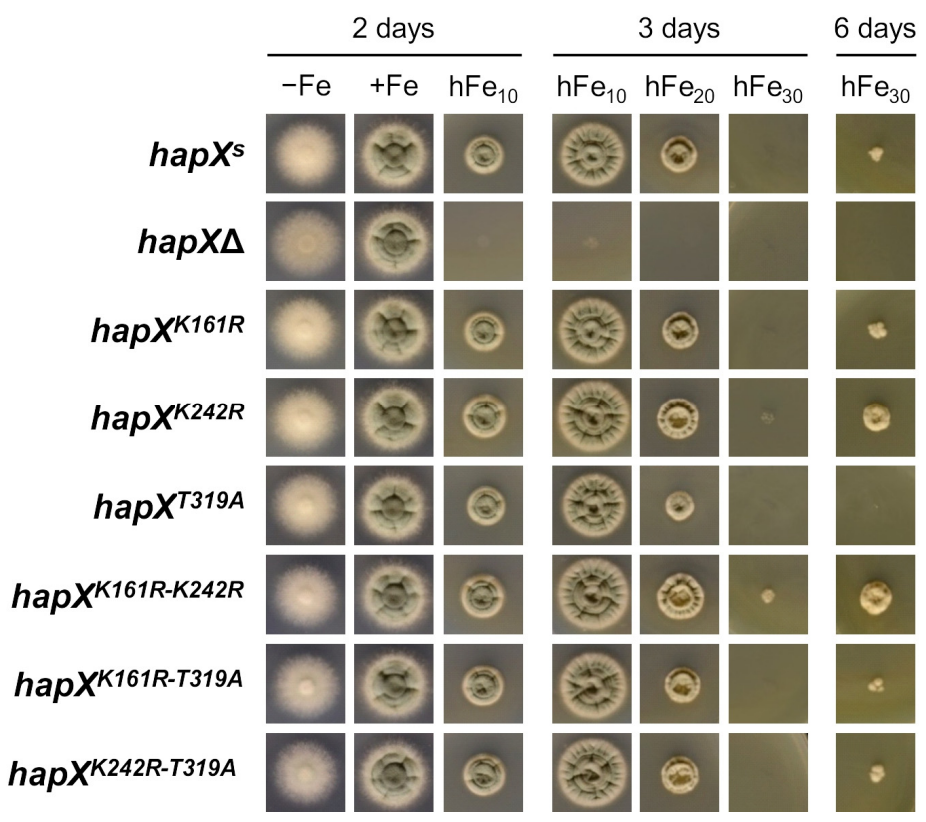

Figure 7. Mutations in HapX K161, K242 and T319 affect iron resistance. Growth of the indicated strains on solid AMM - Fe (iron depletion), +Fe (30 $\left.\mu \mathrm{M} \mathrm{FeSO}_{4}\right)$, and hFe (10, 20 or $\left.30 \mathrm{mM} \mathrm{FeSO}_{4}\right)$. Plates were incubated for the indicated time periods at $37^{\circ} \mathrm{C}$.

Quantification of HapX levels during the adaptation from iron starvation to iron sufficiency, fully comparable between hap $\mathrm{X}^{s}$ and the wild-type strain (compare Figures $2 \mathrm{~B}$ and 8), revealed different patterns in all the tested mutants (Figures 8 and $9 \mathrm{~A}$ ).

In hap $X^{K 161 R},-$ Fe HapX levels were slightly decreased while sFe degradation was similar to that in hap $X^{s}$; In hap $X^{K 242 R}$, like in sumO $\Delta,-$ Fe HapX levels were increased and sFe degradation was slightly less efficient than in hapX $X^{s}$; and in hapX $X^{T 319 A}$, remarkably, -Fe HapX levels were highly increased whereas sFe degradation was tremendously affected in comparison with hap $X^{s}$. Like in the plate assays, the double mutants displayed a HapX degradation pattern consistent with the combination of each of the single mutations (Figure 8). Significantly, the mobility difference observed in HapX 15 min after the iron shift was still detectable in all the mutants (Figure 8). It is important to highlight that in hap $X^{T 319 A}$, HapX levels dropped slightly 15 min after the iron shift and remained stable from then on even when using a higher concentration of iron or a longer incubation time (Figure S4). To exclude the possibility that this phenomenon was due to an alteration of hapX transcriptional regulation, we measured transcript levels of hapX, and of the aforementioned HapX-regulated genes mirB, sidF, sidH, sreA, cycA, cccA and hem $A$, in hap $X^{s}$ and hap $X^{T 319 A}$ during sFe conditions. Expression of hapX was very similar in both strains and, with minor differences, they displayed a transcriptional response characteristic for the adaptation from iron-depleted to iron-replete conditions (Figures S5 and S6). Among the iron-repressed genes, it is remarkable that transcription of sidF and sidH was lower in hap $X^{\mathrm{T} 319 A}$ vs. hap $\mathrm{X}^{s}$ in -Fe (Figure S5) while, in general, iron-induced genes, such as sre $A, \operatorname{cyc} A, \operatorname{ccc} A$ and hem $A$ were more strongly activated in hap $X^{T 319 A}$ vs. hap $X^{s}$ under sFe conditions (Figure S6). A detailed comparative analysis of all the -Fe samples again showed that HapX levels were affected by the combination of each of the single mutations (Figure 9A). Thus, the production of PpIX decreased both in hap $X^{K 242 R}$ and hap $X^{T 319 A}$, and 
in any double mutant with one of these two mutations (Figure 9B). Importantly, HapX migrates as a lower molecular mass band in hap $\mathrm{X}^{\mathrm{K} 161 R}$, and in any double mutant with this mutation, indicating that K161 is post-translationally modified in - Fe conditions (Figure 9A). Taken together, these results show that ubiquitination of K161, sumoylation of K242 and phosphorylation of T319 are important post-translational modifications influencing $\mathrm{hFe}$ resistance, HapX protein levels and production of iron-related metabolites.
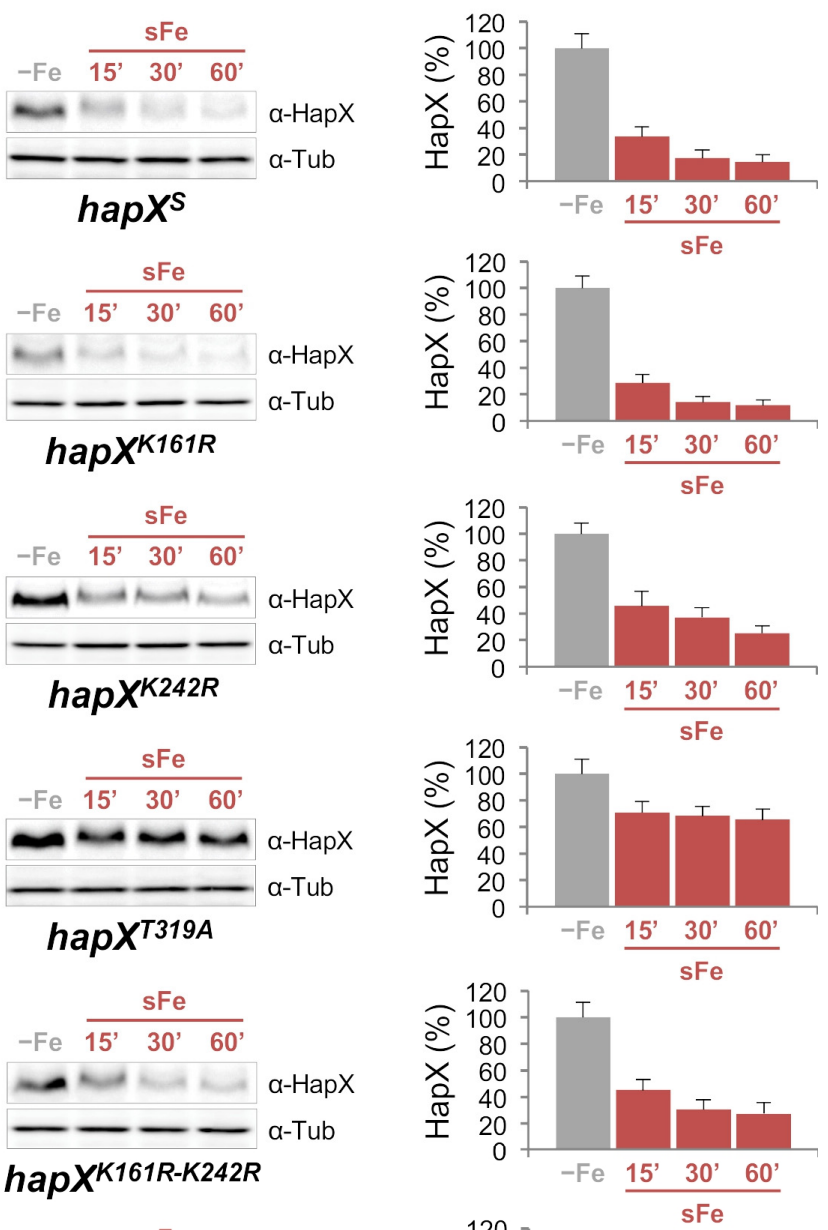

hapXK161R-K242R
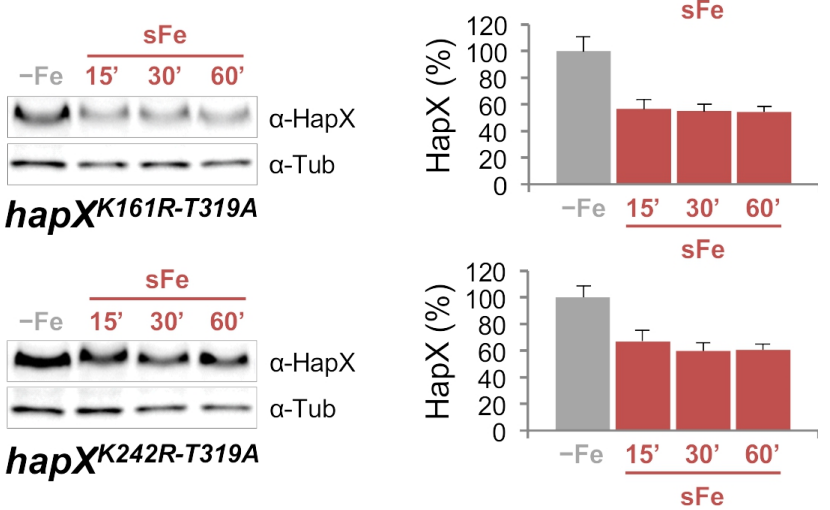

Figure 8. Mutations in HapX K161, K242 and T319 affect HapX post-translational regulation. HapX quantification by Western blot analysis. Samples were obtained from the indicated strains grown under sFe conditions as in Figure 2B. Left panels: Representative Western blot analysis showing HapX protein levels. $\alpha$-Tubulin was used as loading control. Right panels: Densitometric protein quantification. HapX protein levels were normalized to Tubulin and expressed relative to those in $-\mathrm{Fe}$. Bars represent standard deviations from two independent biological experiments with two technical replicates each. 
A
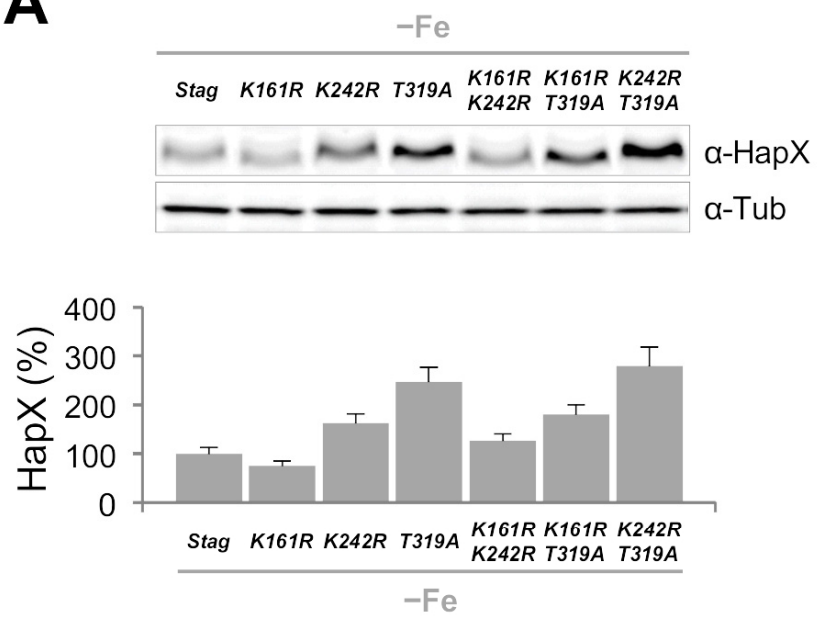

B

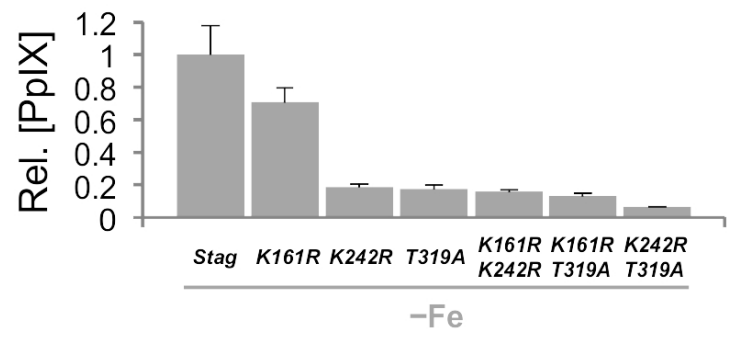

Figure 9. K242 and T319 are important in the regulation of HapX protein levels. (A). HapX quantification by Western blot analysis. Samples were obtained from the indicated strains grown under -Fe conditions as in Figure 2A. Upper panel: Representative Western blot analysis showing HapX protein levels. $\alpha$-Tubulin was used as loading control. Lower panel: Densitometric protein quantification. HapX protein levels were normalized to Tubulin and expressed relative to those in -Fe. (B). Quantification of PpIX in -Fe conditions. PpIX levels were normalized to the amount of total protein and expressed relative to those in hap $X^{s}$. Bars represent standard deviations from two independent biological experiments with two technical replicates each.

\subsection{Overexpression of hapX Negatively Affects Growth and Production of Iron-Related Metabolites}

To analyze the impact of the concentration of HapX in A. fumigatus, we generated a strain in which hapX is under the control of the xylose inducible promoter $x y l P^{P}$ [37], denoted here as strain hap $X^{O E}$ (see materials and methods). Quantitative real-time RT-PCR verified that hapX expression was controlled by the xylose concentration and not the iron status in hap $X^{O E}$ (Figure 10A). In accordance with the tight regulation of HapX observed during sFe conditions, overexpression of the gene $(0.5 \%$ xylose) resulted in growth defects in + Fe cultures (Figure 10B). Slight hapX induction $(0.05 \%$ xylose) gave rise to a wild-type like growth both under $-\mathrm{Fe}$ and $\mathrm{hFe}$ conditions, whereas large overexpression $(0.5$ and $1 \%$ xylose) was detrimental particularly under hFe conditions (Figure S7). This data again underlines the importance of strictly regulating HapX levels for iron homeostasis. Next, we measured accumulation of PpIX in hap $\mathrm{X}^{\mathrm{OE}}$ and, as expected, it was comparable to that in hapX $\Delta$ in the absence of xylose, and 10-fold reduced under overexpression conditions (Figure 10C). Collectively, these results show that increasing the HapX concentration provokes a severe impact on fungal growth and production of iron-related metabolites, highlighting the relevance of modulating HapX levels under different iron conditions. 
A

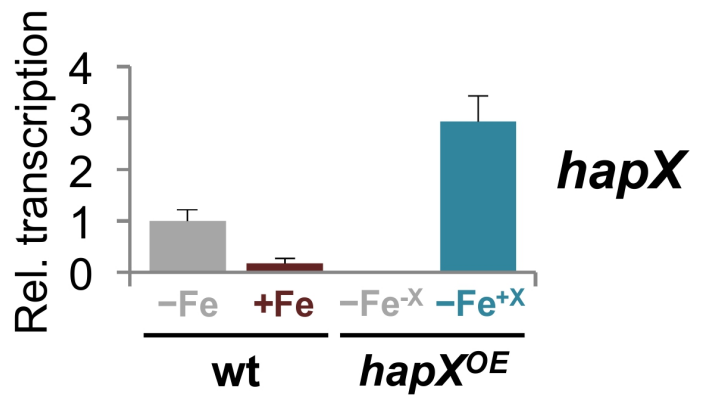

B
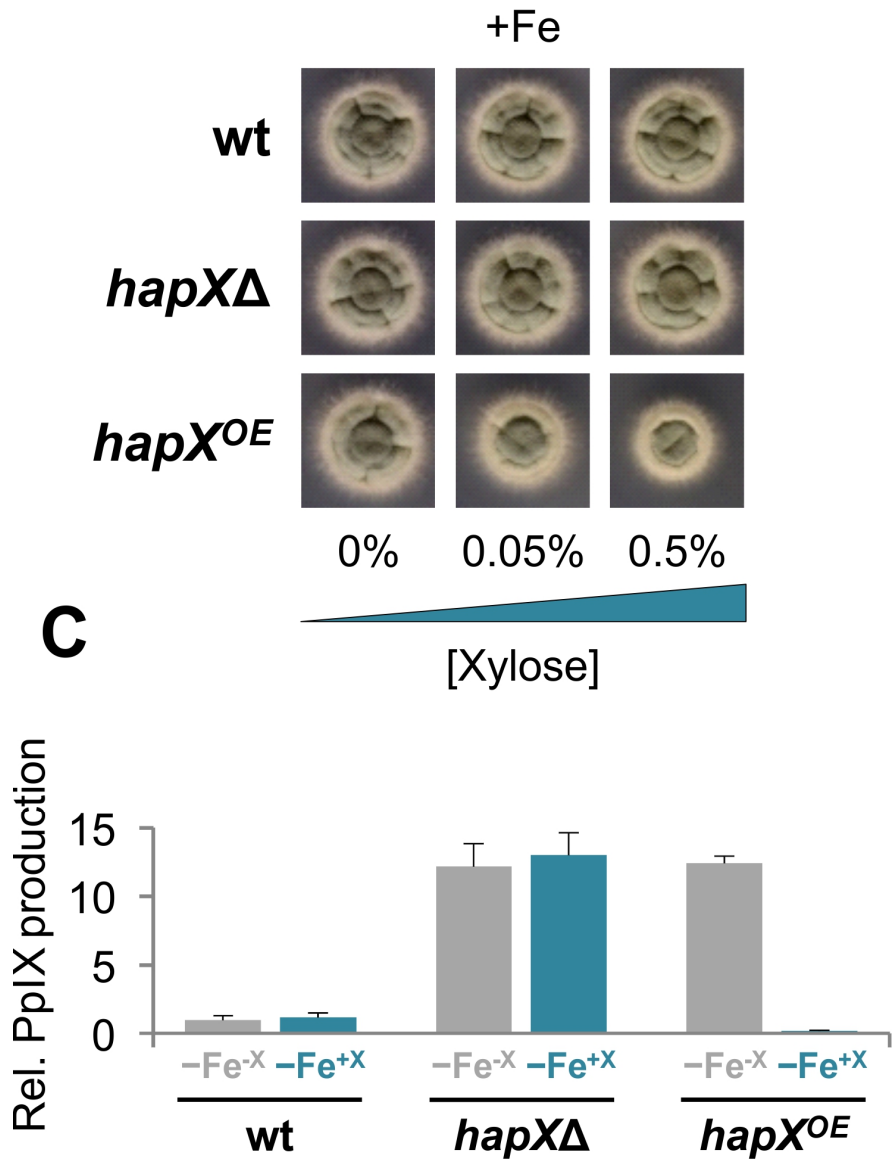

Figure 10. Overexpression of hapX affects growth and PpIX production. (A). Quantitative realtime reverse transcription RT-qPCR. Samples were obtained from the wild-type strain grown under $-\mathrm{Fe}$ and $+\mathrm{Fe}$ conditions as in Figure $2 \mathrm{~A}$, and from hap $\mathrm{X}^{O E}$ grown in $-\mathrm{Fe}$ conditions as in Figure $2 \mathrm{~A}$ $\left(-\mathrm{Fe}^{-\mathrm{X}}\right)$ and then supplemented with $0.01 \%$ xylose for $15 \mathrm{~min}\left(-\mathrm{Fe}^{+\mathrm{X}}\right)$. Transcript levels of the indicated genes, normalized to $a c t A$, are expressed relative to those obtained in the wild-type strain in - Fe. Bars represent standard deviations from two independent biological experiments with two technical replicates each. (B). Growth of the indicated strains on solid AMM $+\mathrm{Fe}\left(30 \mu \mathrm{M} \mathrm{FeSO}_{4}\right)$ with the indicated concentration of xylose (\%). Plates were incubated for $2 \mathrm{~d}$ at $37^{\circ} \mathrm{C}$. (C). Quantification of PpIX in - Fe conditions with or without $0.05 \%$ xylose. PpIX levels of the indicated strains were normalized to the amount of total protein and expressed relative to those in the wild-type strain in the absence of xylose. Bars represent standard deviations from two independent biological experiments with two technical replicates each. 


\section{Discussion}

The CBC:HapX complex, discovered in the early 2000s [38] and molecularly characterized a few years later [20], is essential for the adaptation to different iron availability in filamentous fungi and yeasts [21,22,39-42]. Because HapX-mediated adaptation to iron deficiency is essential for virulence both in animal and plant pathogens [11-15], the study of this bZIP transcription factor has aroused a lot of interest. HapX is required for transcriptional activation of genes involved in iron acquisition as well as for repression of genes implicated in iron-dependent pathways $[11,12,14,16]$. Moreover, HapX was shown to be also essential for the adaptation to iron excess [17]. HapX CRR-A and CRR-B are crucial for this response while the C-terminal domain is responsible for the adaptation to iron starvation [17] (Figure 1). Over the past two decades, a lot of effort has been put into the investigation of HapX mode of action, transcriptional regulation and iron-sensing mechanism $[18,19,21,23,39]$; however, little is known about its regulation at the protein level. Here, we found that there is considerable post-translational regulation of HapX. To gain this insight, we studied A. fumigatus HapX regulation during short-term adaptation of from iron-depleted to iron-replete conditions, identifying new HapX interactors and posttranslational modifications that will break a new scientific ground in our understanding of the regulation of this important transcription factor.

\subsection{HapX Is Regulated at the Protein Level}

HapX was rapidly degraded during sFe conditions (Figure 2B) while the extracellular siderophore biosynthetic enzymes SidF and $\mathrm{SidH}$, whose coding genes are transcriptionally co-regulated with hapX, were not (Figure S2B). Importantly, we noticed that at early time points after the iron shift, HapX migrated as a protein of higher molecular mass (Figures 2B and $3 \mathrm{~B}$ ), which indicates the existence of an iron-dependent post-translational modification mechanism. Furthermore, regardless of this iron-dependent regulation, we show that the half-live of HapX under -Fe conditions is also very short (Figure 3A), indicating that this transcription factor is subject to a complex regulation at the protein level. Protein levels within cells are determined by synthesis and degradation rates with half-lives varying greatly. It is known that many proteins with rapid turnover rates, required to allow their levels to change quickly in response to a stimulus, function as regulatory molecules, such as transcription factors [43]. Our results indicate that HapX is a clear example for such highly controlled regulatory proteins. This finding, together with the previously characterized tight transcriptional regulation [11,20], illustrates the necessity to regulate this transcription factor at multiple levels to strictly control iron homeostasis. MG132-mediated proteasome inhibition [26] prevented HapX degradation during sFe conditions (Figure 2C), which indicates that HapX is degraded, at least partially, in a proteasome dependent manner. It is important to note that the efficiency of the treatment with MG132 is transitory in A. fumigatus most likely due to the presence of multiple genes encoding ATP-binding cassette multidrug transporters in its genome. In yeast, it is known that inactivation of the drug efflux pump PDR5 increases sensitivity to MG132 [44].

\subsection{HapX Interacts with Fbx22 and SumO and Is Prost-Translationally Modified Specifically under sFe Conditions}

Our ${ }^{\mathrm{M}}$ HapX Myc-Trap affinity purification assay followed by nLC-MS/MS-based quantification of the most enriched proteins during sFe conditions identified, apart from the previously reported monothiol glutaredoxin GrxD [18], two uncharacterized HapX interactors, Fbx22 and SumO, mainly in extracts from early time points after iron supplementation (Figure 4). In line with these results two HapX peptides, one containing $\mathrm{K}_{161}{ }^{(\mathrm{GG})}$, a ubiquitin remnant, and another containing $\mathrm{K}_{242}$ (QIGG), a sumoylation remnant, were co-enriched when eluates were digested with the Trypsin/LysC mixture and GluC, respectively (Figure 6A,B). This agrees with the unsuitability of using Trypsin/LysC for MS/MS detection of sumoylated peptides (Figure S8) and GluC for detection of ubiquitinated peptides due to the large size of the remaining attached peptides [45]. Notably, our 
amino acid sequence alignment of the C-terminal region of Aspergillus species orthologs (Figure S8) revealed that except $A$. nidulans and $A$. terreus SumO, all other mature SumO proteins lack a short $C$-terminal extension of amino acids that has to be removed by SUMO peptidases prior to covalent substrate binding via the $\mathrm{C}$-terminal diglycine residue motif. This fact has already been noticed for A. flavus SumO [46].

The F-box protein Fbx22, recently found to be involved in carbon catabolite repression responses in the model fungus Neurospora crassa [47], is a putative ortholog of Saccharomyces cerevisiae Cdc4p, required for G1/S and G2/M phase transitions [48]. Since iron is known to be a major regulator of the cell cycle with both situations, iron limitation and iron excess, related to cell cycle arrest [49], and considering the effect of MG132 on HapX degradation, the HapX-Fbx22 interaction perfectly fits with our experimental data: Fbx22 would interact with HapX, preferentially in the short-term adaptation from iron starvation to iron sufficiency, to promote ubiquitination of the latter and its subsequent degradation, thereby preventing excessive iron accumulation in the cell. Indeed, HapX degradation is delayed in $f b x 22 \Delta$ vs. the wild-type strain under sFe conditions (Figure 5B), but it is still possible. This, along with the observation that HapX still maintains iron-dependent post-translational modification (note the higher molecular mass HapX band immediately after iron addition), indicates that additional mechanisms/players are regulating this process (see below). Furthermore, HapX -Fe content was higher in $f b x 22 \Delta$ vs. the wild-type strain, consequently affecting PpIX production (Figure 5C,D). This finding suggests that Fbx22 plays a role in HapX turnover under - Fe conditions. HapX degradation during sFe conditions was very similar in hap $X^{K 161 R}$ vs. its reference strain $\left(\right.$ hap $\left.X^{S}\right)$ (Figure 8). It is possible that, instead of signaling HapX for degradation, ubiquitination of K161 plays a role in regulation of the protein structure, protein-protein interaction and/or DNA/chromatin interaction. Although we did not detect any additional ubiquitinated lysines in HapX, such assumption is plausible and perhaps the reason why we did not observe changes in the HapX sFe degradation pattern in hap $\mathrm{X}^{\mathrm{K} 161 R}$. In line, it is known that several F-box proteins, such as Cdc4, can ubiquitinate their target proteins on numerous lysines [50] and that interfering with one in particular may have no major impact. Although K161 is preferentially ubiquitinated at early time points after iron supplementation (Figure 6A), this residue is already post-translationally modified under $-\mathrm{Fe}$ conditions. Note that Hap $X^{\mathrm{K} 161 \mathrm{R}}$ migrates as a protein of lower molecular mass in comparison with Hap $\mathrm{X}^{\mathrm{S}}$ (Figure 9A). The fact that the size of HapX is similar in $f b x 22 \Delta$ and the wild-type strain under -Fe conditions (Figure 5C) indicates that Fbx22 is not required for -Fe post-translational modification of K161. Whether K161 is not ubiquitinated via Fbx22, or whether Fbx22 can be replaced by another Fbx protein in $f b x 22 \Delta$ needs to be clarified. Importantly, inactivation of CDC4/Fbx22 is lethal in yeast [51], which might indicate that $\mathrm{Fbx} 22$ functions can be fulfilled by alternative $\mathrm{Fbx}$ proteins in filamentous fungi. On the other hand, we cannot rule out the possibility that HapX is ubiquitinated at other lysine residues via Fbx22. Interestingly, fbx22 $\Delta$ and hap $\mathrm{X}^{\mathrm{K} 161 R}$ are both slightly more resistant to $\mathrm{hFe}$ conditions than their reference strains (Figures 5A and 7) suggesting that the role of Fbx22 and the ubiquitination of HapX K161 are functionally connected.

Transcription factors are among the most frequently detected targets of sumoylation [52,53]. Unlike in S. cerevisiae, where inactivation of SUMO is lethal [34], sumO can be deleted in Aspergilli and Schizosaccharomyces pombe giving rise to drastic phenotypical changes $[33,34,54]$. The relevance of HapX sumoylation is underlined by the fact that both sum $O \Delta$ and hap $X^{K 242 R}$ were more resistant to $\mathrm{hFe}$ conditions (Figures $5 \mathrm{~A}$ and 7). Furthermore, HapX degradation was slightly less efficient during sFe conditions (Figures $5 \mathrm{~B}$ and 8 ), while $-\mathrm{Fe}$ HapX levels were increased, resulting in reduced PpIX accumulation (Figures 5C,D and 9), in both strains. Consistent with the finding that sumoylation often decreases transcription factor-chromatin association to regulate gene expression [52], our results indicate that sumoylation reduces HapX activity. It is important to note that HapX mobility during -Fe conditions was comparable between $\operatorname{sumO} \Delta$ and the wild-type strain and between hap $X^{K 242 R}$ and hap $X^{S}$ (Figures 5C and 9A). This indicates that, unlike K161 ubiquitina- 
tion, sumoylation of K242 occurs almost exclusively under sFe conditions. Interestingly, hap $X^{K 161 R-K 242 R}$, a non-K161-ubiquitinable and non-K242-sumoylatable strain, displayed an intermediate phenotype between those observed for each of the single mutations regarding HapX -Fe content and mobility (Figure 9A) and resistance to hFe concentrations (Figure 7). Therefore, both signals independently regulate HapX function in A. fumigatus.

Besides ubiquitination and sumoylation, we found that HapX T319 is phosphorylated preferentially under sFe conditions (Figure 6C). Replacement of HapX T319 with alanine dramatically increased HapX - Fe content (Figures 8 and 9A), thereby causing decreased PpIX accumulation (Figure 9B), even though hapX transcript levels during iron starvation were similar in hapX ${ }^{\mathrm{T} 319 A}$ vs. its reference strain (Figure S5). Furthermore, HapX ${ }^{\mathrm{T} 319 \mathrm{~A}}$ degradation during sFe conditions was extraordinarily diminished (Figure 8 and Figure S4). These results indicate that phosphorylation of T319 is essential for the maintenance of the HapX turnover rate and for HapX degradation under sFe conditions. Moreover, tolerance to $\mathrm{hFe}$ concentration was reduced in hap $\mathrm{X}^{\mathrm{T} 319 A}$ (Figure 7) highlighting the relevance of regulating the level of HapX in response to different iron conditions. In agreement with this observation, overexpression of hapX negatively affected $A$. fumigatus growth under

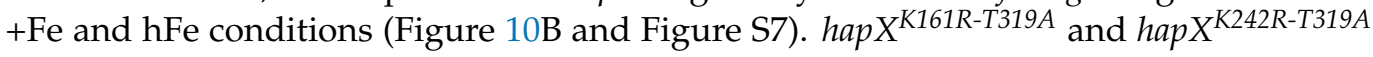
exhibited a mixture of each of the individual mutations concerning HapX -Fe levels and mobility (Figure 9A), HapX degradation during sFe conditions (Figure 8), and resistance to $\mathrm{hFe}$ concentrations (Figure 7). This suggests that regulation of HapX via phosphorylation of T319 acts independently of K161 ubiquitination and K242 sumoylation in A. fumigatus. Whether there is crosstalk between the detected reversible post-translational modifications or between these and others that we may have not detected to regulate HapX function and stability remains to be elucidated. For example, it is known that phosphorylation can promote ubiquitination that can lead to proteasomal degradation [55]. The effect of MG132 treatment and T319A substitution on HapX degradation during sFe conditions points towards this direction. Considering all our results, we postulate that K161 ubiquitination and K242 sumoylation mainly regulate HapX activity while T319 phosphorylation regulates HapX stability.

One important question is how it is mechanistically possible that these residues, K161, K242 and T319, are modified within such a short time period. Because HapX is itself an iron sensor, most likely via [2Fe-2S] cluster binding [18,19], it is possible that the modifying apparatus recognizes the conformational change that HapX undergoes upon binding of [2Fe-2S] clusters. This conformational change could make the recognition motifs harboring K161, K242 and T319 more accessible for the respective modifications. In any case, this study clearly demonstrates that HapX is subject to complex post-translational modifications in A. fumigatus.

\section{Materials and Methods}

\subsection{Fungal Isolates and Culture Conditions}

Aspergillus fumigatus AfS77 (akuAD strain derived from ATCC46645 lacking nonhomologous recombination) was used in all experiments [56] cultured at $37^{\circ} \mathrm{C}$ for the indicated time periods. Fungal strains were stored as conidial suspensions at $-80^{\circ} \mathrm{C}$ with $30 \%$ glycerol. All strains used in this study are listed in Table S1. For liquid cultures, $10^{6}$ conidia $\mathrm{mL}^{-1}$ were cultured at $200 \mathrm{rpm}$ in Aspergillus minimal medium (AMM) [57] containing $1 \%$ glucose $(w / v)$ and $20 \mathrm{mM}$ glutamine as carbon and nitrogen sources, respectively. For solid cultures, $2 \times 10^{4}$ conidia were spotted onto AMM agar or agarose (to avoid iron contaminations) plates. Iron $\left(\mathrm{FeSO}_{4}\right)$ concentrations used in each experiment are indicated in the figures. To inhibit the proteasome, MG132 (Merck KGaA, Darmstadt, Germany) was used at a final concentration of $50 \mu \mathrm{M}$. To block protein biosynthesis, $50 \mu \mathrm{g} \cdot \mathrm{mL}^{-1}$ of the translation inhibitor cycloheximide (chx) (Merck KGaA, Darmstadt, Germany) we used. In hap $X^{O E}$, hapX transcription was induced with the indicated concentrations of xylose $(w / v)$. 


\subsection{Generation of Mutant Strains}

For venus-tagging of sidF and sidH ( ${ }^{v}$ sidF and ${ }^{v}$ sidH), cassettes, including each coding sequence N-terminally linked with the venus-tag under the control of their native promoter and terminator region as well as the hygromycin resistance marker, were assembled by fusion PCR [58] and protoplasts of A. fumigatus AfS77 were transformed to hygromycin resistance.

For myc-tagging of hapX $\left({ }^{m}\right.$ hapX $)$, the cassette, including the hapX coding sequence $\mathrm{N}$-terminally linked with myc-tag under the control of its native promoter and the nosterminator (nosT) region as well as the hygromycin resistance marker, was obtained by Gibson assembly and was performed using the NEBuilder ${ }^{\circledR}$ HiFi DNA Assembly Master Mix (New England Biolabs, Frankfurt, Germany) [59]. The cassette was then used to transform a hapX $\Delta$ strain in which the pyrithiamine resistance gene had been used to replace the hapX coding sequence [17].

For the generation of A. fumigatus fbx22 (Afu6g13030) and sumO (Afu1g10850) deletion mutant strains, targeted replacement cassettes were constructed amplifying DNA fragments flanking both coding sequences and fusing them with the pyrithiamine resistance marker by Gibson assembly [59]. Subsequently, protoplasts of A. fumigatus AfS77 were transformed to pyrithiamine resistance.

For the generation of hap $X^{K 161 R}$, hap $X^{K 242 R}$, hapX $X^{T 319 A}$, hap $X^{K 161 R-K 242 R}$, hap $X^{K 161 R-T 319 A}$ and hap $X^{\mathrm{K} 242 R-T 319 A}$ mutant strains, we used the basic plasmid phap $X^{\mathrm{R}}$-hph [17]. In order to substitute specific amino acids by site-directed mutagenesis, the QuickChange SiteDirected Mutagenesis Kit (Aligent, Santa Clara, CA, USA) was used. For the introduction of each mutation, phap $X^{\mathrm{R}}$-hph was amplified with complementary primers including the desired change. Resulting plasmids were confirmed by sequencing. Plasmids were then linearized and used to transform a hapX $\Delta$ strain in which the pyrithiamine resistance gene had been used to replace the hapX coding sequence [17].

For conditional expression of hapX (hap $\left.X^{O E}\right)$, we generated a plasmid containing the hapX coding sequence C-terminally linked with an S-tag [17] under the control of $x y l P^{P}$, a xylan/xylose-inducible promoter derived from the xylanase $x y l P$ of Penicillium chrysogenum [37], as well as the hygromycin resistance marker. The plasmid was then linearized and used to transform a hapX $\Delta$ strain in which the pyrithiamine resistance gene had been used to replace the hapX coding sequence [17].

In all cases, transformants showing homologous insertions were genotyped by PCR and Southern blot analysis (not shown).

\subsection{Nucleic Acid Manipulations, Quantitative Real-Time RT-PCR and PpIX Analysis}

Total RNA and gDNA were extracted from A. fumigatus mycelia following previously reported protocols [60]. Quality and quantity of extracted nucleic acids were determined by running aliquots in ethidium bromide-stained agarose gels and by spectrophotometric analysis in a NanoDrop ND-1000 spectrophotometer (NanoDrop Technologies, Wilmington, DE, USA), respectively. Quantitative RT-PCR was performed as described previously [12,61] using FastStart Essential DNA Green Master (Roche Diagnostics SL, Barcelona, Spain) in a CFX Connect Real-Time System (Bio-Rad, Madrid, Spain). Gene specific primers were designed to flank an intron, if possible. Transcript levels were calculated by comparative $\Delta C t$ and normalized to $a c t A$. PpIX production analysis was carried out as described previously [20].

\subsection{Western Blotting}

Proteins were extracted using a reported procedure [62] involving solubilization from lyophilized mycelial biomass with $\mathrm{NaOH}$, followed by precipitation with trichloroacetic acid (TCA). Aliquots were resolved in 10-12\% SDS-polyacrylamide gels (Carl Roth GmbH + Co. KG, Karlsruhe, Germany) and transferred to nitrocellulose membranes with a Trans-Blot Turbo Transfer System (Bio-Rad, Madrid, Spain) for blotting. Western blots were reacted with anti-HapX antisera (1:20,000; Davids Biotechnologie, Regensburg, Germany) or with 
polyclonal anti-GFP (1:10,000; A11122 ThermoFisher Scientific Inc., Waltham, MA, USA) as primary antibodies and with monoclonal anti-rabbit IgG peroxidase (1:10,000; A1949 Merck KGaA, Darmstadt, Germany) as secondary antibody. Tubulin, used as loading control, was detected with monoclonal anti- $\alpha$-tubulin (1:10,000; T6119 Merck KGaA, Darmstadt, Germany) as primary antibody and with polyclonal anti-mouse IgG peroxidase (1:10,000; A4416 Merck KGaA, Darmstadt, Germany) as secondary antibody. Proteins were detected with ECL (Thermo Fisher Scientific, Waltham, MA, USA) and densitometric quantifications were performed with ImageJ 1.48v (National Institutes of Health, Bethesda, MD, USA).

\subsection{Myc-Trap Immunoprecipitation of ${ }^{M}$ HapX Fusion Protein}

A. fumigatus mycelia were harvested in Stop buffer [63] at $4{ }^{\circ} \mathrm{C}$ after growth for $22 \mathrm{~h}$ and freeze-dried. Protein extraction was performed according to a modified procedure from [63] using HK buffer containing $10 \mu \mathrm{M} N$-Ethylmaleimide (NEM) (Merck KGaA, Darmstadt, Germany) for total protein extraction. All steps were carried out at $4{ }^{\circ} \mathrm{C}$ in the cold room. In brief, $100 \mathrm{mg}$ of freeze-dried mycelium powder were ground in a MixerMill MM 400 (Retsch GmbH, Haan, Germany) for $1 \mathrm{~min}$ at $30 \mathrm{~Hz}$ and then digested again in $1 \mathrm{~mL}$ HK buffer for $1 \mathrm{~min}$ at $30 \mathrm{~Hz}$. Cell extracts were centrifuged twice at 20,187 $\times g$ for $15 \mathrm{~min}$ and $500 \mu \mathrm{L}$ of the supernatant was incubated with Myc-Trap ${ }^{\circledR}$ agarose beads (ChromoTek GmbH, Planegg-Martinsried, Germany) for $1 \mathrm{~h}$. The beads were washed twice in HK buffer without NP40, twice in wash buffer $(25 \mathrm{mM}$ Tris/ $\mathrm{HCl} \mathrm{pH} \mathrm{7.5,} 500 \mathrm{mM} \mathrm{NaCl}$, $5 \mathrm{mM}$ EDTA and $15 \mathrm{mM}$ EGTA) and once in ultrapure water. Proteins were eluted in $10 \%$ $(v / v)$ acetonitrile and $5 \%(v / v)$ acetic acid and used for nLC-MS/MS measurement and Western blot detection.

\subsection{In-Solution Digest of Myc-Trap Eluates}

Dried Myc-Trap eluates were solubilized in $50 \mu \mathrm{L} 50 \mathrm{mM} \mathrm{NH}_{4} \mathrm{HCO}_{3}$ in 50:50 (v/v) trifluoroethanol (TFE)/water. After heat denaturation $\left(90^{\circ} \mathrm{C}, 10 \mathrm{~min}\right)$ the proteins were reduced for $1 \mathrm{~h}$ at $55^{\circ} \mathrm{C}$ by addition of TCEP (Tris(2-carboxyethyl)phosphine) at a final concentration of $8 \mathrm{mM}$. Further carbamidomethylation was performed for $45 \mathrm{~min}$ at $32{ }^{\circ} \mathrm{C}$ in $15 \mathrm{mM}$ chloroacetamide. Subsequently the samples were evaporated in a vacuum concentrator (Eppendorf, Wesseling, Germany) to a residual volume of approximately $5 \mu \mathrm{L}$. Finally, the volume was set to $30 \mu \mathrm{L}$ with $50 \mathrm{mM} \mathrm{NH} 4 \mathrm{HCO} 3$ and proteins were digested overnight $\left(18 \mathrm{~h}, 37^{\circ} \mathrm{C}\right)$ with a Trypsin/LysC mixture (Promega, Walldorf, Germany) as well as GluC endoproteinase (Promega, Walldorf, Germany) at a protein to protease ratio of $25: 1$. The digestion was stopped by adding $10 \%(v / v)$ formic acid and peptides were dried in a vacuum concentrator (Eppendorf, Wesseling, Germany), re-solubilized in $20 \mu \mathrm{L}$ of $0.05 \%$ TFA in $\mathrm{H}_{2} \mathrm{O}$ /acetonitrile $98 / 2(v / v)$ and filtered through a $10 \mathrm{kDa}$ spin filter. The filtrate was transferred to HPLC vials and injected into the LC-MS/MS instrument. Each sample was measured in triplicate (3 analytical replicates).

\section{7. $n L C-M S / M S$ Analysis}

nLC-MS/MS analysis was carried out on an Ultimate 3000 nano (n) RSLC system coupled to a QExactive Plus mass spectrometer (both Thermo Fisher Scientific, Waltham, MA, USA). Peptides were trapped for $5 \mathrm{~min}$ on an Acclaim Pep Map 100 column $(2 \mathrm{~cm} \times 75 \mu \mathrm{m}$, $3 \mu \mathrm{m})$ at $5 \mu \mathrm{L} / \mathrm{min}$ followed by gradient elution separation on an Acclaim Pep Map RSLC column $(50 \mathrm{~cm} \times 75 \mu \mathrm{m}, 2 \mu \mathrm{m})$. Eluent A $(0.1 \%(v / v)$ formic acid in water) was mixed with eluent $\mathrm{B}(0.1 \%(v / v)$ formic acid in $90 / 10$ acetonitrile/water) as follows: $0 \mathrm{~min}$ at $4 \% \mathrm{~B}$, $6 \mathrm{~min}$ at $6 \% \mathrm{~B}, 14 \mathrm{~min}$ at $10 \% \mathrm{~B}, 20 \mathrm{~min}$ at $14 \% \mathrm{~B}, 35 \mathrm{~min}$ at $20 \% \mathrm{~B}, 42 \mathrm{~min}$ at $26 \% \mathrm{~B}, 46 \mathrm{~min}$ at $32 \% \mathrm{~B}, 52 \mathrm{~min}$ at $42 \% \mathrm{~B}, 55 \mathrm{~min}$ at $50 \% \mathrm{~B}, 58 \mathrm{~min}$ at $65 \% \mathrm{~B}, 60-64.9 \mathrm{~min}$ at $96 \% \mathrm{~B}, 65-90 \mathrm{~min}$ at $4 \%$ B. Positively charged ions were generated at $2.2 \mathrm{kV}$ using a stainless steel emitter and a Nanospray Flex Ion Source (Thermo Fisher Scientific, Waltham, MA, USA). The QExactive Plus was operated in Full MS/data-dependent MS2 (Top10) mode. Precursor ions were monitored at $m / z 300-1500$ at a resolution of 70,000 FWHM (full width at half maximum) using a maximum injection time (ITmax) of $120 \mathrm{~ms}$ and an AGC (automatic 
gain control) target of $1 \times 10^{6}$. Precursor ions with a charge state of $\mathrm{z}=2-5$ were filtered at an isolation width of $m / z 1.6$ amu for HCD fragmentation at 30\% normalized collision energy (NCE). MS2 ions were scanned at 17,500 FWHM (ITmax $\left.=120 \mathrm{~ms}, \mathrm{AGC}=2 \times 10^{5}\right)$. Dynamic exclusion of precursor ions was set to $20 \mathrm{~s}$. The LC-MS/MS instrument was controlled by QExactive Plus Tune 2.9 and Xcalibur 3.0 with DCMS Link.

\subsection{Protein Database Search}

Tandem mass spectra were searched against the Aspergillus Genome Database (AspGD) of Aspergillus fumigatus Af293 (http:/ / www.aspergillusgenome.org/download/sequence/ A_fumigatus_Af293/current/A_fumigatus_Af293_current_orf_trans_all.fasta.gz (accessed on 4 February 2019)) and the protein sequence of Dre2 (AFUB_008090; the Dre2 ortholog is not present in the Af293 gene annotation) as well as further modified protein sequences (e.g., Myc-tag) using Proteome Discoverer (PD) 2.2 (ThermoFisher Scientific Inc., Waltham, MA, USA) and the algorithms of Sequest HT (version of PD2.2) and MS Amanda 2.0. Two missed cleavages were allowed for the proteolytic digestions. The precursor mass tolerance was set to $10 \mathrm{ppm}$ and the fragment mass tolerance was set to $0.02 \mathrm{Da}$. Modifications were defined as dynamic oxidation of Met, acetylation of Ser, phosphorylation of Ser, Thr, and Tyr, ubiquitination (GG) and sumoylation (QIGG) of Lys as well as static Cys carbamidomethylation. At least two peptides per protein and a strict false discovery rate $($ FDR $)<1 \%$ were required for positive protein hits. The Percolator node of PD2.2 and a reverse decoy database was used for q-value validation of spectral matches. Only rank 1 proteins and peptides of the top scored proteins were counted. The Minora algorithm of PD2.2 was applied for relative label-free quantification. Myc-Trap eluates from wt A. fumigatus mycelial extracts were used for quantification of nonspecifically co-purified proteins. Potential HapX-interacting proteins were determined using the two-sided $t$-test and visualized by volcano plots.

\subsection{Western Blot Detection of Proteins after Myc-Trap}

Proteins were separated by SDS-PAGE using NuPAGE ${ }^{\mathrm{TM}} 4-12 \%(w / v)$ Bis-Tris gradient gels (ThermoFisher Scientific Inc., Waltham, MA, USA). For Western detection, proteins were transferred onto a PVDF membrane using the iBlot ${ }^{\mathrm{TM}} 2$ dry blotting system (ThermoFisher Scientific Inc., Waltham, MA, USA). The membrane was blocked in 3\% $(w / v)$ bovine serum albumin (BSA) dissolved in 1x PBST (137 mM NaCl, $2.7 \mathrm{mM} \mathrm{KCl}, 10 \mathrm{mM}$ $\mathrm{Na}_{2} \mathrm{HPO}_{4}, 2 \mathrm{mM} \mathrm{KH} \mathrm{PO}_{4}, 0.05 \%(v / v)$ Tween 20). Western blots were reacted with antiHapX antisera (1:10,000; Davids Biotechnologie, Regensburg, Germany) or with polyclonal anti-c-Myc (1:1000; ab9106 Abcam plc, Cambridge, UK) as primary antibodies and with monoclonal anti-rabbit IgG peroxidase (1:10,000; A1949 Merck KGaA, Darmstadt, Germany) as secondary antibody. The membrane was developed using the 1-Step ${ }^{\mathrm{TM}}$ Ultra TMB-Blotting chromogenic substrate (ThermoFisher Scientific Inc., Waltham, MA, USA).

Supplementary Materials: Figure S1. Transcription of iron-regulated genes. Figure S2. SidF and SidH are stable during sFe conditions. Figure S3. Effective enrichment of ${ }^{\mathrm{M}} \mathrm{HapX}$ validated by Western blot and nLC-MS/MS analysis. Figure S4. Quantification of HapX in hap $X^{S}$ and hap $X^{T 319 A}$ under different sFe conditions. Figure S5. Transcription of iron-repressed genes. Figure S6. Transcription of iron-induced genes. Figure S7. Effect of HapX overexpression on -Fe and hFe colony growth. Figure S8. Amino acid sequence alignment of the C-terminal region of SumO orthologs from various Aspergillus species. Table S1. Strains used in this study.

Author Contributions: Conceptualization, M.S.L.-B., M.T.S., P.H., A.A.B. and H.H.; Methodology, M.S.L.-B., M.T.S., P.H., M.M., C.B., F.G., E.R.W., T.K., J.W., O.K., A.A.B. and H.H.; Software, M.S.L.-B., M.T.S., P.H., T.K., J.W.; Validation, M.S.L.-B., M.T.S., P.H., M.M., C.B., F.G., E.R.W., T.K., J.W., O.K., A.A.B. and H.H.; Formal analysis, M.S.L.-B., M.T.S., P.H., M.M., C.B., F.G., E.R.W., T.K., J.W. and H.H.; Investigation, M.S.L.-B., M.T.S., P.H., M.M., C.B., F.G., E.R.W., T.K., J.W., O.K., A.A.B. and H.H.; Resources, M.S.L.-B., P.H., T.K., A.A.B. and H.H.; Data curation, M.S.L.-B., P.H., T.K. and H.H.; Writing—original draft preparation, M.S.L.-B., P.H. and H.H.; Writing-review and editing, M.S.L.-B., M.T.S., P.H., M.M., C.B., F.G., T.K., J.W., A.A.B. and H.H.; Visualization, M.S.L.-B., M.T.S., P.H., 
M.M., C.B., F.G., E.R.W., T.K., J.W., O.K., A.A.B. and H.H.; Supervision, M.S.L.-B., P.H., T.K., O.K., A.A.B. and H.H.; Project administration, M.S.L.-B., P.H., O.K., A.A.B. and H.H.; Funding acquisition, M.S.L.-B., P.H., O.K., A.A.B. and H.H. All authors have read and agreed to the published version of the manuscript.

Funding: This research was funded by the Austrian Science Fund (FWF), grant number M1962 B-21, and by the Junta de Andalucía, grant number 27375-R, to M.S.L.B. Moreover, this work was partially funded by the Joint D-A-CH program 'Novel molecular mechanisms of iron sensing and homeostasis in filamentous fungi' (https:/ / gepris.dfg.de/gepris/projekt/241377596 (accessed on 16 July 2021)) from the Austrian Science Fund (FWF) I1346-B22 to H.H. and the Deutsche Forschungsgemeinschaft (DFG) BR 1130/14-1 to A.A.B., by the DFG HO 2596/1-1 to P.H., by the Euregio Science Fund (SupErA IPN95) to H.H., and by the DFG CRC/TR 124 FungiNet (project number 210879364, project Z2) to O.K.

Institutional Review Board Statement: Not applicable.

Informed Consent Statement: Not applicable.

Data Availability Statement: The mass spectrometry proteomics data have been deposited to the ProteomeXchange Consortium via the PRIDE [64] partner repository with the dataset identifier PXD026889.

Acknowledgments: We are grateful to Sylke Fricke, María Ortega Bellido and Beate Abt for excellent technical assistance.

Conflicts of Interest: The authors declare no conflict of interest. The sponsors had no role in the design, execution, interpretation, or writing of the study.

$\begin{array}{ll}\text { Abbreviations } \\ \text { RIA } & \text { Reductive iron assimilation } \\ \text { CBC } & \text { CCAAT-binding complex } \\ \text { CRR } & \text { Cysteine rich region } \\ -\mathrm{Fe} & \text { Iron-deplete } \\ +\mathrm{Fe} & \text { Iron-replete } \\ \text { sFe } & \text { Shift from iron-depleted to iron-replete } \\ \text { FsC } & \text { Fusarinine C } \\ \text { TAFC } & \text { Triacetylfusarinine C } \\ \text { chx } & \text { Cycloheximide } \\ \text { LFQ } & \text { Label-free quantification abundances } \\ \text { SCF } & \text { Skp, Cullin, F-box } \\ \text { hFe } & \text { high iron } \\ \text { PpIX } & \text { Protoporphyrin IX } \\ \text { NDSM } & \text { Negatively charged amino acid-dependent SUMOylation motif } \\ \text { AMM } & \text { Aspergillus minimal medium } \\ \text { TCA } & \text { Trichloroacetic acid }\end{array}$

\section{References}

1. De Hoog, G.S.; Guarro, J. Atlas of Clinical Fungi; Centraalbureau voor Schimmelcultures: Utrecht, The Netherlands, 1995.

2. Tekaia, F.; Latge, J.P. Aspergillus fumigatus: Saprophyte or pathogen? Curr. Opin. Microbiol. 2005, 8, 385-392. [CrossRef] [PubMed]

3. Brown, G.D.; Denning, D.W.; Gow, N.A.; Levitz, S.M.; Netea, M.G.; White, T.C. Hidden killers: Human fungal infections. Sci. Transl. Med. 2012, 4, 165rv13. [CrossRef] [PubMed]

4. Bongomin, F.; Gago, S.; Oladele, R.O.; Denning, D.W. Global and Multi-National Prevalence of Fungal Diseases-Estimate Precision. J. Fungi 2017, 3, 57. [CrossRef] [PubMed]

5. Brakhage, A.A. Systemic fungal infections caused by Aspergillus species: Epidemiology, infection process and virulence determinants. Curr. Drug Targets 2005, 6, 875-886. [CrossRef] [PubMed]

6. Fisher, M.C.; Henk, D.A.; Briggs, C.J.; Brownstein, J.S.; Madoff, L.C.; McCraw, S.L.; Gurr, S.J. Emerging fungal threats to animal, plant and ecosystem health. Nature 2012, 484, 186-194. [CrossRef] [PubMed]

7. Calo, S.; Shertz-Wall, C.; Lee, S.C.; Bastidas, R.J.; Nicolas, F.E.; Granek, J.A.; Mieczkowski, P.; Torres-Martinez, S.; Ruiz-Vazquez, R.M.; Cardenas, M.E.; et al. Antifungal drug resistance evoked via RNAi-dependent epimutations. Nature 2014, 513, 555-558. [CrossRef] 
8. Robbins, N.; Caplan, T.; Cowen, L.E. Molecular Evolution of Antifungal Drug Resistance. Annu. Rev. Microbiol. $2017,71,753-775$. [CrossRef] [PubMed]

9. Halliwell, B.; Gutteridge, J.M.C. Oxygen-toxicity, oxygen radicals, transition-metals and disease. Biochem. J. 1984, 219 , 1-14. [CrossRef]

10. Lin, H.; Li, L.; Jia, X.; Ward, D.M.; Kaplan, J. Genetic and biochemical analysis of high iron toxicity in yeast: Iron toxicity is due to the accumulation of cytosolic iron and occurs under both aerobic and anaerobic conditions. J. Biol. Chem. 2011, 286, 3851-3862. [CrossRef]

11. Schrettl, M.; Beckmann, N.; Varga, J.; Heinekamp, T.; Jacobsen, I.D.; Joechl, C.; Moussa, T.A.; Wang, S.; Gsaller, F.; Blatzer, M.; et al. HapX-Mediated Adaption to Iron Starvation Is Crucial for Virulence of Aspergillus fumigatus. PLoS Pathog. 2010, 6, e1001124. [CrossRef]

12. Lopez-Berges, M.S.; Capilla, J.; Turra, D.; Schafferer, L.; Matthijs, S.; Joechl, C.; Cornelis, P.; Guarro, J.; Haas, H.; Di Pietro, A. HapX-mediated iron homeostasis is essential for rhizosphere competence and virulence of the soilborne pathogen Fusarium oxysporum. Plant Cell 2012, 24, 3805-3822. [CrossRef]

13. Hsu, P.-C.; Yang, C.-Y.; Lan, C.-Y. Candida albicans Hap43 is a repressor induced under low-iron conditions and is essential for iron-responsive transcriptional regulation and virulence. Eukaryot. Cell 2011, 10, 207-225. [CrossRef] [PubMed]

14. Jung, W.H.; Saikia, S.; Hu, G.; Wang, J.; Fung, C.K.-Y.; D’Souza, C.; White, R.; Kronstad, J.W. HapX Positively and Negatively Regulates the Transcriptional Response to Iron Deprivation in Cryptococcus neoformans. PLoS Pathog. 2010, 6, e1001209. [CrossRef] [PubMed]

15. Wang, Y.; Deng, C.; Tian, L.; Xiong, D.; Tian, C.; Klosterman, S.J. The Transcription Factor VdHapX Controls Iron Homeostasis and Is Crucial for Virulence in the Vascular Pathogen Verticillium dahliae. Msphere 2018, 3, e00400-18. [CrossRef] [PubMed]

16. Schrettl, M.; Kim, H.S.; Eisendle, M.; Kragl, C.; Nierman, W.C.; Heinekamp, T.; Werner, E.R.; Jacobsen, I.; Illmer, P.; Yi, H.; et al. SreA-mediated iron regulation in Aspergillus fumigatus. Mol. Microbiol. 2008, 70, 27-43. [CrossRef]

17. Gsaller, F.; Hortschansky, P.; Beattie, S.R.; Klammer, V.; Tuppatsch, K.; Lechner, B.E.; Rietzschel, N.; Werner, E.R.; Vogan, A.A.; Chung, D.; et al. The Janus transcription factor HapX controls fungal adaptation to both iron starvation and iron excess. EMBO J. 2014, 33, 2261-2276. [CrossRef]

18. Misslinger, M.; Scheven, M.T.; Hortschansky, P.; Lopez-Berges, M.S.; Heiss, K.; Beckmann, N.; Heigl, T.; Hermann, M.; Kruger, T.; Kniemeyer, O; ; et al. The monothiol glutaredoxin GrxD is essential for sensing iron starvation in Aspergillus fumigatus. PLoS Genet. 2019, 15, e1008379. [CrossRef]

19. Misslinger, M.; Lechner, B.E.; Bacher, K.; Haas, H. Iron-sensing is governed by mitochondrial, not by cytosolic iron-sulfur cluster biogenesis in Aspergillus fumigatus. Met. Integr. Biometal Sci. 2018, 10, 1687-1700. [CrossRef]

20. Hortschansky, P.; Eisendle, M.; Al-Abdallah, Q.; Schmidt, A.D.; Bergmann, S.; Thon, M.; Kniemeyer, O.; Abt, B.; Seeber, B.; Werner, E.R.; et al. Interaction of HapX with the CCAAT-binding complex-A novel mechanism of gene regulation by iron. Embo J. 2007, 26,3157-3168. [CrossRef]

21. Hortschansky, P.; Haas, H.; Huber, E.M.; Groll, M.; Brakhage, A.A. The CCAAT-binding complex (CBC) in Aspergillus species. Biochim. Biophys. Acta Gene Regul. Mech. 2017, 1860, 560-570. [CrossRef] [PubMed]

22. Furukawa, T.; Scheven, M.T.; Misslinger, M.; Zhao, C.; Hoefgen, S.; Gsaller, F.; Lau, J.; Jochl, C.; Donaldson, I.; Valiante, V.; et al. The fungal CCAAT-binding complex and HapX display highly variable but evolutionary conserved synergetic promoter-specific DNA recognition. Nucleic Acids Res. 2020, 48, 3567-3590. [CrossRef] [PubMed]

23. Schrettl, M.; Haas, H. Iron homeostasis-Achilles' heel of Aspergillus fumigatus? Curr. Opin. Microbiol. 2011, 14, 400-405. [CrossRef] [PubMed]

24. Schrettl, M.; Bignell, E.; Kragl, C.; Sabiha, Y.; Loss, O.; Eisendle, M.; Wallner, A.; Arst, H.N., Jr.; Haynes, K.; Haas, H. Distinct roles for intra- and extracellular siderophores during Aspergillus fumigatus infection. PLoS Pathog. 2007, 3, e128. [CrossRef] [PubMed]

25. Yasmin, S.; Alcazar-Fuoli, L.; Gruendlinger, M.; Puempel, T.; Cairns, T.; Blatzer, M.; Lopez, J.F.; Grimalt, J.O.; Bignell, E.; Haas, H. Mevalonate governs interdependency of ergosterol and siderophore biosyntheses in the fungal pathogen Aspergillus fumigatus. Proc. Natl. Acad. Sci. USA 2012, 109, E497-E504. [CrossRef]

26. Lee, D.H.; Goldberg, A.L. Selective inhibitors of the proteasome-dependent and vacuolar pathways of protein degradation in Saccharomyces cerevisiae. J. Biol. Chem. 1996, 271, 27280-27284. [CrossRef]

27. Schneider-Poetsch, T.; Ju, J.; Eyler, D.E.; Dang, Y.; Bhat, S.; Merrick, W.C.; Green, R.; Shen, B.; Liu, J.O. Inhibition of eukaryotic translation elongation by cycloheximide and lactimidomycin. Nat. Chem. Biol. 2010, 6, 209-217. [CrossRef] [PubMed]

28. Deshpande, I.; Keusch, J.J.; Challa, K.; Iesmantavicius, V.; Gasser, S.M.; Gut, H. The Sir4 H-BRCT domain interacts with phospho-proteins to sequester and repress yeast heterochromatin. EMBO J. 2019, 38, e101744. [CrossRef]

29. Skowyra, D.; Craig, K.L.; Tyers, M.; Elledge, S.J.; Harper, J.W. F-box proteins are receptors that recruit phosphorylated substrates to the SCF ubiquitin-ligase complex. Cell 1997, 91, 209-219. [CrossRef]

30. Jonkers, W.; Rep, M. Lessons from fungal F-box proteins. Eukaryot Cell 2009, 8, 677-695. [CrossRef]

31. de Assis, L.J.; Ulas, M.; Ries, L.N.A.; El Ramli, N.A.M.; Sarikaya-Bayram, O.; Braus, G.H.; Bayram, O.; Goldman, G.H. Regulation of Aspergillus nidulans CreA-Mediated Catabolite Repression by the F-Box Proteins Fbx23 and Fbx47. MBio 2018, 9, e00840-18. [CrossRef]

32. Welchman, R.L.; Gordon, C.; Mayer, R.J. Ubiquitin and ubiquitin-like proteins as multifunctional signals. Nat. Rev. Mol. Cell Biol. 2005, 6, 599-609. [CrossRef] [PubMed] 
33. Harting, R.; Bayram, O.; Laubinger, K.; Valerius, O.; Braus, G.H. Interplay of the fungal sumoylation network for control of multicellular development. Mol. Microbiol. 2013, 90, 1125-1145. [CrossRef] [PubMed]

34. Wong, K.H.; Todd, R.B.; Oakley, B.R.; Oakley, C.E.; Hynes, M.J.; Davis, M.A. Sumoylation in Aspergillus nidulans: sumO inactivation, overexpression and live-cell imaging. Fungal Genet. Biol. 2008, 45, 728-737. [CrossRef]

35. Sahu, M.S.; Patra, S.; Kumar, K.; Kaur, R. SUMOylation in Human Pathogenic Fungi: Role in Physiology and Virulence. J. Fungi 2020, 6, 32. [CrossRef] [PubMed]

36. Yang, S.H.; Galanis, A.; Witty, J.; Sharrocks, A.D. An extended consensus motif enhances the specificity of substrate modification by SUMO. EMBO J. 2006, 25, 5083-5093. [CrossRef]

37. Zadra, I.; Abt, B.; Parson, W.; Haas, H. xylP promoter-based expression system and its use for antisense downregulation of the Penicillium chrysogenum nitrogen regulator NRE. Appl. Environ. Microbiol. 2000, 66, 4810-4816. [CrossRef]

38. Tanaka, A.; Kato, M.; Nagase, T.; Kobayashi, T.; Tsukagoshi, N. Isolation of genes encoding novel transcription factors which interact with the Hap complex from Aspergillus species. Biochim. Biophys. Acta Gene Struct. Expr. 2002, 1576, 176-182. [CrossRef]

39. Misslinger, M.; Hortschansky, P.; Brakhage, A.A.; Haas, H. Fungal iron homeostasis with a focus on Aspergillus fumigatus. Biochim. Biophys. Acta Mol. Cell Res. 2021, 1868, 118885. [CrossRef]

40. Bairwa, G.; Hee Jung, W.; Kronstad, J.W. Iron acquisition in fungal pathogens of humans. Met. Integr. Biometal Sci. 2017, 9, 215-227. [CrossRef]

41. Gerwien, F.; Skrahina, V.; Kasper, L.; Hube, B.; Brunke, S. Metals in fungal virulence. FEMS Microbiol. Rev. 2018, 42, fux050. [CrossRef]

42. Labbe, S.; Khan, M.G.; Jacques, J.F. Iron uptake and regulation in Schizosaccharomyces pombe. Curr. Opin. Microbiol. 2013, 16, 669-676. [CrossRef] [PubMed]

43. Cooper, G.M. The Cell: A Molecular Approach, 8th ed.; Oxford University Press: New York, NY, USA, 2019.

44. Collins, G.A.; Gomez, T.A.; Deshaies, R.J.; Tansey, W.P. Combined chemical and genetic approach to inhibit proteolysis by the proteasome. Yeast 2010, 27, 965-974. [CrossRef]

45. Impens, F.; Radoshevich, L.; Cossart, P.; Ribet, D. Mapping of SUMO sites and analysis of SUMOylation changes induced by external stimuli. Proc. Natl. Acad. Sci. USA 2014, 111, 12432-12437. [CrossRef] [PubMed]

46. Nie, X.; Yu, S.; Qiu, M.; Wang, X.; Wang, Y.; Bai, Y.; Zhang, F.; Wang, S. Aspergillus flavus SUMO Contributes to Fungal Virulence and Toxin Attributes. J. Agric. Food Chem. 2016, 64, 6772-6782. [CrossRef]

47. Horta, M.A.C.; Thieme, N.; Gao, Y.; Burnum-Johnson, K.E.; Nicora, C.D.; Gritsenko, M.A.; Lipton, M.S.; Mohanraj, K.; de Assis, L.J.; Lin, L.; et al. Broad Substrate-Specific Phosphorylation Events Are Associated with the Initial Stage of Plant Cell Wall Recognition in Neurospora crassa. Front. Microbiol. 2019, 10, 2317. [CrossRef] [PubMed]

48. Hartwell, L.H. Genetic control of the cell division cycle in yeast. II. Genes controlling DNA replication and its initiation. J. Mol. Biol. 1971, 59, 183-194. [CrossRef]

49. Zhang, C. Essential functions of iron-requiring proteins in DNA replication, repair and cell cycle control. Protein Cell 2014, 5, 750-760. [CrossRef] [PubMed]

50. Sadowski, M.; Suryadinata, R.; Tan, A.R.; Roesley, S.N.; Sarcevic, B. Protein monoubiquitination and polyubiquitination generate structural diversity to control distinct biological processes. IUBMB Life 2012, 64, 136-142. [CrossRef]

51. Giaever, G.; Chu, A.M.; Ni, L.; Connelly, C.; Riles, L.; Veronneau, S.; Dow, S.; Lucau-Danila, A.; Anderson, K.; Andre, B.; et al. Functional profiling of the Saccharomyces cerevisiae genome. Nature 2002, 418, 387-391. [CrossRef]

52. Rosonina, E.; Akhter, A.; Dou, Y.; Babu, J.; Sri Theivakadadcham, V.S. Regulation of transcription factors by sumoylation. Transcription 2017, 8, 220-231. [CrossRef]

53. Boulanger, M.; Chakraborty, M.; Tempe, D.; Piechaczyk, M.; Bossis, G. SUMO and Transcriptional Regulation: The Lessons of Large-Scale Proteomic, Modifomic and Genomic Studies. Molecules 2021, 26, 828. [CrossRef] [PubMed]

54. Tanaka, K.; Nishide, J.; Okazaki, K.; Kato, H.; Niwa, O.; Nakagawa, T.; Matsuda, H.; Kawamukai, M.; Murakami, Y. Characterization of a fission yeast SUMO-1 homologue, pmt3p, required for multiple nuclear events, including the control of telomere length and chromosome segregation. Mol. Cell Biol. 1999, 19, 8660-8672. [CrossRef]

55. Hunter, T. The age of crosstalk: Phosphorylation, ubiquitination, and beyond. Mol. Cell 2007, 28, 730-738. [CrossRef] [PubMed]

56. Hartmann, T.; Dumig, M.; Jaber, B.M.; Szewczyk, E.; Olbermann, P.; Morschhauser, J.; Krappmann, S. Validation of a self-excising marker in the human pathogen Aspergillus fumigatus by employing the beta-rec/six site-specific recombination system. Appl. Environ. Microbiol. 2010, 76, 6313-6317. [CrossRef] [PubMed]

57. Pontecorvo, G.; Roper, J.A.; Hemmons, L.M.; Macdonald, K.D.; Bufton, A.W. The genetics of Aspergillus nidulans. Adv. Genet. 1953, 5, 141-238.

58. Szewczyk, E.; Nayak, T.; Oakley, C.E.; Edgerton, H.; Xiong, Y.; Taheri-Talesh, N.; Osmani, S.A.; Oakley, B.R. Fusion PCR and gene targeting in Aspergillus nidulans. Nat. Protoc. 2006, 1, 3111-3120. [CrossRef] [PubMed]

59. Gibson, D.G.; Young, L.; Chuang, R.Y.; Venter, J.C.; Hutchison, C.A., 3rd; Smith, H.O. Enzymatic assembly of DNA molecules up to several hundred kilobases. Nat. Methods 2009, 6, 343-345. [CrossRef]

60. Sambrook, J.; Russell, D. Molecular Cloning: A Laboratory Manual, 3rd ed.; Cold Spring Harbour Laboratory Press: New York, NY, USA, 2001.

61. Lopez-Berges, M.S.; Rispail, N.; Prados-Rosales, R.C.; Di Pietro, A. A Nitrogen Response Pathway Regulates Virulence Functions in Fusarium oxysporum via the Protein Kinase TOR and the bZIP Protein MeaB. Plant Cell 2010, 22, 2459-2475. [CrossRef] 
62. Hervas-Aguilar, A.; Penalva, M.A. Endocytic machinery protein SlaB is dispensable for polarity establishment but necessary for polarity maintenance in hyphal tip cells of Aspergillus nidulans. Eukaryot Cell 2010, 9, 1504-1518. [CrossRef]

63. Liu, H.L.; Osmani, A.H.; Ukil, L.; Son, S.; Markossian, S.; Shen, K.F.; Govindaraghavan, M.; Varadaraj, A.; Hashmi, S.B.; De Souza, C.P.; et al. Single-step affinity purification for fungal proteomics. Eukaryot Cell 2010, 9, 831-833. [CrossRef]

64. Perez-Riverol, Y.; Csordas, A.; Bai, J.; Bernal-Llinares, M.; Hewapathirana, S.; Kundu, D.J.; Inuganti, A.; Griss, J.; Mayer, G.; Eisenacher, M.; et al. The PRIDE database and related tools and resources in 2019: Improving support for quantification data. Nucleic Acids Res. 2019, 47, D442-D450. [CrossRef] [PubMed] 